\title{
Multigranular Uncertain Linguistic Prioritized Aggregation Operators and Their Application to Multiple Criteria Group Decision Making
}

\author{
Ding-Hong Peng, ${ }^{1,2}$ Tie-Dan Wang, ${ }^{1}$ Chang-Yuan Gao, ${ }^{2}$ and Hua Wang ${ }^{1}$ \\ ${ }^{1}$ Institute of Quality Development, Kunming University of Science and Technology, Kunming 650093, China \\ ${ }^{2}$ School of Management, Harbin University of Science and Technology, Harbin 150040, China \\ Correspondence should be addressed to Ding-Hong Peng; pengdinghong2009@163.com
}

Received 23 January 2013; Accepted 20 March 2013

Academic Editor: Frank Werner

Copyright (C) 2013 Ding-Hong Peng et al. This is an open access article distributed under the Creative Commons Attribution License, which permits unrestricted use, distribution, and reproduction in any medium, provided the original work is properly cited.

\begin{abstract}
We investigate multiple criteria group decision-making problems in which there are priority relationships between the decision elements (criteria and experts), and decision information provided by decision makers takes the form of multigranular uncertain linguistic information. Firstly, some operational laws and possibility degree of multi-granular uncertain linguistic variables are introduced. Then, some new linguistic aggregation operators based on the prioritized aggregation operator, such as the multigranular uncertain linguistic prioritized weighted average (MULPWA) operator and the multigranular uncertain linguistic prioritized ordered weighted average (MULPOWA) operator, are developed and their desirable properties are studied. The prominent characteristics of these proposed operators are that they can aggregate directly the uncertain linguistic variables whose values form the linguistic term sets with different granularities and convey the prioritization phenomenon among the aggregated arguments. Furthermore, based on the MULPWA and MULPOWA operators, an approach to deal with multiple criteria group decision-making problems under multi-granular uncertain linguistic environments is developed. Finally, a practical example is provided to illustrate the multiple criteria group decision-making process.
\end{abstract}

\section{Introduction}

Due to the fact that experiences and judgments of humans are usually represented by words in their natural language, decision making with linguistic information is becoming a hot research topic and has received many excellent results in recent years [1-35]. When using linguistic approaches to solve decision problems, we need the techniques for computing with words (CWW). In the specialized literature, the main linguistic computational models can be divided into four kinds: the approximate model based on extension principle [1]; the ordered language model [2]; the 2-tuple model [8], and the virtual linguistic variables model [9]. Compared with the former two models, the latter two models can avoid losing any linguistic information moreover, Dong et al. $[11,12]$ proved that the latter two models can be mutually retranslated, and they are equivalent.
However, in many situations, the decision information is expressed in the form of uncertain linguistic variables which can be considered as intervals of linguistic terms because of time pressure, lack of knowledge or data, and limited expertise related to the problem domain. Therefore, $\mathrm{Xu}$ [13-16] presented the concept of uncertain linguistic variables, and then various uncertain linguistic aggregation operators have been proposed, such as the uncertain linguistic averaging (ULA) operator, uncertain linguistic weighted averaging (ULWA) operator, uncertain linguistic ordered weighted averaging (ULOWA) operator, uncertain linguistic hybrid aggregation (ULHA) operator, uncertain linguistic geometric mean (ULGM) operator, uncertain linguistic weighted geometric mean (ULWGM) operator, uncertain linguistic ordered weighted geometric (ULOWG) operator, uncertain linguistic hybrid geometric mean (ULHG) operator [17], induced uncertain linguistic OWA (IULOWA), induced uncertain 
linguistic ordered weighted geometric(IULOWG) operator, uncertain linguistic correlated averaging (ULCA) operator, uncertain linguistic correlated geometric (ULCG) operator, and uncertain linguistic harmonic mean (ULHM) operators [18]. More recently, Zhang [19] proposed the interval-valued 2-tuple linguistic variable and introduced some aggregation operators of interval-valued 2-tuples. Chen and Lee [20] proposed interval linguistic labels ordered weighted average (ILLOWA) operator for autocratic decision making. Chen et al. [21] presented an adaptive consensus support model for group decision making systems based on intervals of linguistic 2-tuples.

On the other hand, due to the difference in culture, cognition, experience, habits, backgrounds, and so on, decision makers may express their evaluation using different linguistic domains with different granularities, that is, multigranular linguistic term sets, in practical decision making problems. Up to now, many approaches have been proposed to deal with GDM problems with multigranular linguistic information [22-32]. In these approaches, the multigranularity linguistic terms are first unified into a basic linguistic term set (BLTS) by transform functions; usually the BLTS is linguistic term set with the greatest granularity, before the linguistic information aggregation. Zhang [19] pointed out that the transform processes are tedious and may produce the loss of information and proposed interval-valued 2-tuple linguistic variable to directly aggregate interval-valued 2tuple linguistic information.

Decision making with uncertain linguistic information and with multigranular linguistic information has significantly advanced decision analysis with linguistic information. However, in these studies, the multigranular uncertain linguistic information is seldom addressed [30]. Xu (2009) [28] developed some transformation functions to unify the unbalanced linguistic labels with different granularities and then utilized the uncertain linguistic weighted averaging (ULWA) operator to aggregate the unified unbalanced linguistic information. Fan and Liu (2010) [30] proposed a method with multigranular uncertain linguistic information, in which multigranular uncertain linguistic values are first transformed into trapezoidal fuzzy numbers and then an extended TOPSIS methodology is used to solve the decisionmaking problem. Gao and Peng (2011) [31] defined the transformation function of uncertain linguistic preferences with different granularities. Zhang and Guo (2012) [32] proposed a method for multigranular uncertain linguistic group decision making with incomplete weight information. Meanwhile, current linguistic decision making methods are under the assumption that the criteria are at the same priority level, and they are characterized by the ability to trade off between criteria. However, in lots of real and practical multiple criteria decision-making problems, the criteria have different priority levels commonly. Such as in the case of buying a car based upon the criteria of safety and cost, usually we may not allow compensation between cost and safety, and in this case of organizational decision making, superiors generally have a higher priority than those of their subordinates. Yager [36] paid attention to this issue and presented the prioritized aggregation operators by modeling the prioritization of attributes with respect to the weights associated with the attributes dependent upon the satisfaction of the higher priority attributes. Afterwards, some authors devoted their attentions to this issue [37-41]. However, to our knowledge, no study has reported an appropriate method for solving the prioritization phenomenon among the criteria using linguistic information, letting alone the problems with multigranular uncertain linguistic information. Therefore, it is necessary to pay attention to this issue.

To do this, the remainder of this paper is organized as follows. Section 2 introduces the operational laws of multigranular uncertain linguistic variables and briefly reviews the prioritized aggregation operators. Section 3 proposes the multigranular uncertain linguistic prioritized weighted average (MULPWA) operator and multigranular uncertain linguistic prioritized ordered weighted average (MULPOWA) operator to aggregate the multigranular uncertain linguistic variables, whose desirable properties are also studied in this section. In Section 4, we develop an approach for multicriteria group decision making based on the proposed operators under multigranular uncertain linguistic environment. In Section 5, a practical example is provided to verify the practicality and effectiveness of the developed approach. Section 6 concludes the paper.

\section{Preliminaries}

2.1. Uncertain Linguistic Variables. Let $S^{(g)}=\left\{s_{i} \mid i=\right.$ $0,1, \ldots, g-1\}$ be a linguistic term set with odd cardinality, where $s_{i}$ represents a possible value for a linguistic variable and $g$ denote the granularity of the term set, and it usually has the following characteristics [1-5]: (1) the set is ordered: $s_{i} \geq s_{j}$ if $i \geq j$; (2) there is the negation operator: $\operatorname{neg}\left(s_{i}\right)=s_{j}$ such that $j=g-1-i$; (3) max operator: $\max \left(s_{i}, s_{j}\right)=s_{i}$ if $s_{i} \geq s_{j}$; (4) min operator: $\min \left(s_{i}, s_{j}\right)=s_{i}$ if $s_{i} \leq s_{j}$. For example, a linguistic term set with 9 terms $S$ can be defined as $S^{(9)}=\left\{s_{0}=\right.$ extremely poor, $s_{1}=$ very poor, $s_{2}=$ poor, $s_{3}=$ slightly poor, $s_{4}=$ fair, $s_{5}=$ slightly good, $s_{6}=$ good, $s_{7}=$ very good, $s_{8}=$ extremely good $\}$.

Usually, the granularity $g$ of $S^{(g)}$ must be small enough so as not to impose useless precision on the experts and it must be rich enough in order to allow a discrimination of the performances of each object in a limited number of grades; the limit of cardinality is 11 or not more than $13[3,4]$.

To preserve all the given information, $\mathrm{Xu}[9,10]$ extended the discrete term set $S^{(g)}$ to a continuous term set $\bar{S}^{(g)}=\left\{s_{\alpha} \mid\right.$ $\left.s_{0} \leq s_{\alpha} \leq s_{g-1}, \alpha \in[0, g-1]\right\}$, whose elements also meet all the characteristics above, and if $s_{\alpha} \in S$, then we call $s_{\alpha}$ the original term; otherwise, we call $s_{\alpha}$ the virtual term.

Definition 1 (see $[13,14]$ ). Let $\widetilde{s}=\left[s_{\alpha}, s_{\beta}\right]$, where $s_{\alpha}, s_{\beta} \in \bar{S}$, and $s_{\alpha}$ and $s_{\beta}$ are the lower and the upper limits, respectively; we then call $\widetilde{s}$ the uncertain linguistic variable.

In the existing literature, the operational laws of uncertain linguistic variables are all assumed that the values of uncertain linguistic variables are from the same linguistic term set. So they may be not fit to the uncertain linguistic information 
from multigranular linguistic term sets, that is, the multigranular uncertain linguistic information. In order to process the multigranular uncertain linguistic information, we give the following definitions.

Definition 2. For any three uncertain linguistic variables $\widetilde{s}^{(g)}=\left[s_{\alpha}^{(g)}, s_{\beta}^{(g)}\right], \widetilde{s}_{1}^{\left(g_{1}\right)}=\left[s_{\alpha_{1}}^{\left(g_{1}\right)}, s_{\beta_{1}}^{\left(g_{1}\right)}\right]$, and $\widetilde{s}_{2}^{\left(g_{2}\right)}=\left[s_{\alpha_{2}}^{\left(g_{2}\right)}, s_{\beta_{2}}^{\left(g_{2}\right)}\right]$ and their linguistic values from linguistic term sets with granularities $g, g_{1}$, and $g_{2}$, respectively, then their operational laws can be defined as follows:

$$
\begin{aligned}
&(1) \mu \widetilde{s}^{(g)}= {\left[\mu s_{\alpha}^{(g)}, \mu s_{\beta}^{(g)}\right]=\left[s_{\mu \alpha}^{(g)}, s_{\mu \beta}^{(g)}\right], } \\
&(2)\left(\widetilde{s}^{(g)}\right)^{\mu}=\left[\left(s_{\alpha}^{(g)}\right)^{\mu},\left(s_{\beta}^{(g)}\right)^{\mu}\right]=\left[s_{\alpha^{\mu}}^{(g)}, s_{\beta^{\mu}}^{(g)}\right], \\
& \text { (3) } \widetilde{s}_{1}^{\left(g_{1}\right)} \oplus \widetilde{s}_{2}^{\left(g_{2}\right)}=\left[s_{\alpha_{1}}^{\left(g_{1}\right)}, s_{\beta_{1}}^{\left(g_{1}\right)}\right] \oplus\left[s_{\alpha_{2}}^{\left(g_{2}\right)}, s_{\beta_{2}}^{\left(g_{2}\right)}\right] \\
&=\left[\frac{g^{*}-1}{g_{1}-1} s_{\alpha_{1}}^{\left(g_{1}\right)} \oplus \frac{g^{*}-1}{g_{1}-1} s_{\alpha_{2}}^{\left(g_{2}\right)},\right. \\
& \\
&\left.\quad \frac{g^{*}-1}{g_{1}-1} s_{\beta_{1}}^{\left(g_{1}\right)} \oplus \frac{g^{*}-1}{g_{1}-1} s_{\beta_{2}}^{\left(g_{2}\right)}\right], \\
&=\left[s_{\left(\left(g^{*}-1\right) /\left(g_{1}-1\right)\right) \alpha_{1}+\left(\left(g^{*}-1\right) /\left(g_{2}-1\right)\right) \alpha_{2}}^{\left(g^{*}\right)}\right. \\
& s_{\left(\left(g^{*}-1\right) /\left(g_{1}-1\right)\right) \beta_{1}+\left(\left(g^{*}-1\right) /\left(g_{2}-1\right)\right) \beta_{2}}^{\left(g^{*}\right)},
\end{aligned}
$$

(4) $\widetilde{s}_{1}^{\left(g_{1}\right)} \otimes \widetilde{s}_{2}^{\left(g_{2}\right)}=\left[s_{\alpha_{1}}^{\left(g_{1}\right)}, s_{\beta_{1}}^{\left(g_{1}\right)}\right] \otimes\left[s_{\alpha_{2}}^{\left(g_{2}\right)}, s_{\beta_{2}}^{\left(g_{2}\right)}\right]$

$$
\begin{aligned}
= & {\left[\frac{g^{*}-1}{g_{1}-1} s_{\alpha_{1}}^{\left(g_{1}\right)} \otimes \frac{g^{*}-1}{g_{2}-1} s_{\alpha_{2}}^{\left(g_{2}\right)},\right.} \\
& \left.\frac{g^{*}-1}{g_{1}-1} s_{\beta_{1}}^{\left(g_{1}\right)} \otimes \frac{g^{*}-1}{g_{2}-1} s_{\beta_{2}}^{\left(g_{2}\right)}\right] \\
= & {\left[s_{\left(\left(g^{*}\right)\right.}^{\left.\left(g^{*}-1\right) /\left(g_{1}-1\right)\right) \alpha_{1} \times\left(\left(g^{*}-1\right) /\left(g_{2}-1\right)\right) \alpha_{2}},\right.} \\
& \left.s_{\left(\left(g^{*}-1\right) /\left(g_{1}-1\right)\right) \beta_{1} \times\left(\left(g^{*}-1\right) /\left(g_{2}-1\right)\right) \beta_{2}}^{\left(g^{*}\right)}\right],
\end{aligned}
$$

where $g^{*} \in\left\{g_{1}, g_{2}\right\}$ is the granularity of basic term set.

From the operational laws (3) and (4), we can get the following equations:

$$
\begin{aligned}
& \bigoplus_{j=1}^{n} \widetilde{s}_{j}^{\left(g_{j}\right)}=\bigoplus_{j=1}^{n}\left[s_{\alpha_{j}}^{\left(g_{j}\right)}, s_{\beta_{j}}^{\left(g_{j}\right)}\right] \\
& =\left[\bigoplus_{j=1}^{n} \frac{g^{*}-1}{g_{j}-1} s_{\alpha_{1}}^{\left(g_{j}\right)}, \bigoplus_{j=1}^{n} \frac{g^{*}-1}{g_{j}-1} s_{\beta_{j}}^{\left(g_{j}\right)}\right] \\
& =\left[s^{\left(g^{*}\right)}\left(g^{*}-1\right) \sum_{j=1}^{n}\left(\alpha_{j} /\left(g_{j}-1\right)\right),\right.
\end{aligned}
$$

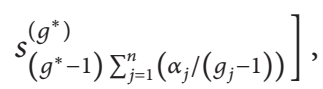

(6)

$$
\begin{aligned}
& \bigoplus_{j=1}^{n} \widetilde{s}_{j}^{\left(g_{j}\right)}=\bigoplus_{j=1}^{n}\left[s_{\alpha_{j}}^{\left(g_{j}\right)}, s_{\beta_{j}}^{\left(g_{j}\right)}\right] \\
& =\left[\bigoplus_{j=1}^{n} \frac{g^{*}-1}{g_{j}-1} s_{\alpha_{1}}^{\left(g_{j}\right)}, \bigoplus_{j=1}^{n} \frac{g^{*}-1}{g_{j}-1} s_{\beta_{1}}^{\left(g_{j}\right)}\right] \\
& =\left[s_{\prod_{j=1}^{n}\left(g^{*}\right)}^{\left(\left(g^{*}-1\right) /\left(g_{j}-1\right)\right) \alpha_{j}}, s_{\prod_{j=1}^{n}\left(\left(g^{*}-1\right) /\left(g_{j}-1\right)\right) \beta_{j}}^{\left(g^{*}\right)}\right] \text {, }
\end{aligned}
$$

where $g^{*} \in\left\{g_{1}, g_{2}, \ldots, g_{n}\right\}$ is the granularity of basic term set.

To compare uncertain linguistic variables, $\mathrm{Xu}$ (2004) [13] proposed a possibility degree formula to calculate an uncertain linguistic term that is greater than the other one. In the following, we further extend the possibility degree formula to compare the multigranular uncertain linguistic variables.

Definition 3. Let $\widetilde{s}_{1}^{\left(g_{1}\right)}=\left[s_{\alpha_{1}}^{\left(g_{1}\right)}, s_{\beta_{1}}^{\left(g_{1}\right)}\right]$ and $\widetilde{s}_{2}^{\left(g_{2}\right)}=\left[s_{\alpha_{2}}^{\left(g_{2}\right)}, s_{\beta_{2}}^{\left(g_{2}\right)}\right]$ be uncertain linguistic variables and their linguistic values from linguistic terms sets with granularities $g_{1}$ and $g_{2}$, respectively, and then the possibility degree $\widetilde{s}_{1}^{g_{1}}$ dominate $\widetilde{s}_{2}^{g_{2}}$ is defined as

$$
\begin{aligned}
P\left(\widetilde{s}_{1}^{\left(g_{1}\right)} \geq\right. & \left.\widetilde{s}_{2}^{\left(g_{2}\right)}\right) \\
= & \left(\max \left\{0,\left(g_{2}-1\right) \beta_{1}-\left(g_{1}-1\right) \alpha_{2}\right\}\right. \\
& \left.-\max \left\{0,\left(g_{2}-1\right) \alpha_{1}-\left(g_{1}-1\right) \beta_{2}\right\}\right) \\
& \times\left(\left(g_{2}-1\right)\left(\beta_{1}-\alpha_{1}\right)+\left(g_{1}-1\right)\left(\beta_{2}-\alpha_{2}\right)\right)^{-1} .
\end{aligned}
$$

If $s_{\alpha_{1}}^{\left(g_{1}\right)}=s_{\beta_{1}}^{\left(g_{1}\right)}$ and $s_{\alpha_{2}}^{\left(g_{2}\right)}=s_{\beta_{2}}^{\left(g_{2}\right)}$, then the possibility degree $\widetilde{s}_{1}^{g_{1}}$ dominate $\widetilde{s}_{2}^{g_{2}}$ is defined as

$$
P\left(\widetilde{s}_{1}\left(g_{1}\right) \geq \widetilde{s}_{2}^{\left(g_{2}\right)}\right)= \begin{cases}1, & \text { if }\left(g_{2}-1\right) s_{\alpha_{1}}^{\left(g_{1}\right)}>\left(g_{1}-1\right) s_{\alpha_{2}}^{\left(g_{2}\right)} \\ 0.5, & \text { if } \left.\left(g_{2}-1\right) s_{\alpha_{1}} g_{1}\right)=\left(g_{1}-1\right) s_{\alpha_{2}}^{\left(g_{2}\right)} \\ 0, & \text { if } \left.\left(g_{2}-1\right) s_{\alpha_{1}} g_{1}\right)<\left(g_{1}-1\right) s_{\alpha_{2}}^{\left(g_{2}\right)} .\end{cases}
$$

From Definition 3, we can easily get the following results:

(1) $0 \leq P\left(\widetilde{s}_{1}^{\left(g_{1}\right)} \geq \widetilde{s}_{2}^{\left(g_{2}\right)}\right) \leq 1$, Especially, $P\left(\widetilde{s}_{1}^{\left(g_{1}\right)} \geq \widetilde{s}_{1}^{\left(g_{1}\right)}\right)=$ 0.5

(2) $P\left(\widetilde{s}_{1}^{\left(g_{1}\right)} \geq \widetilde{s}_{2}^{\left(g_{2}\right)}\right)+P\left(\widetilde{s}_{2}^{\left(g_{2}\right)} \geq \widetilde{s}_{1}^{\left(g_{1}\right)}\right)=1$, especially, $P\left(\widetilde{s}_{1}^{\left(g_{1}\right)} \geq \widetilde{s}_{2}^{\left(g_{2}\right)}\right)=P\left(\widetilde{s}_{2}^{\left(g_{2}\right)} \geq \widetilde{s}_{1}^{\left(g_{1}\right)}\right)=0.5$,

(3) $P\left(\widetilde{s}_{1}^{\left(g_{1}\right)} \geq \widetilde{s}_{2}^{\left(g_{2}\right)}\right)=P\left(\lambda \widetilde{s}_{1}^{\left(g_{1}\right)} \geq \lambda \widetilde{s}_{2}^{\left(g_{2}\right)}\right)$, where $\lambda \epsilon$ $(-\infty, 0) \cup(0,+\infty)$.

Note that if $g_{1}=g_{2}=\cdots=g_{n}$, then the above results reduced to the ones of uncertain linguistic variables [13]. Based on the possibility degree and the idea of [42], the relative possibility degree $P\left(\widetilde{s}_{i}^{\left(g_{i}\right)}\right)$ of $\widetilde{s}_{i}^{\left(g_{i}\right)}$ over all $\widetilde{s}_{j}^{\left(g_{j}\right)}(j=$ $1,2, \ldots, n)$ can be defined as follows:

$$
P\left(\widetilde{s}_{i}^{\left(g_{i}\right)}\right)=\frac{1}{n(n-1)}\left(\sum_{j=1}^{n} P\left(\widetilde{s}_{i}^{\left(g_{i}\right)} \geq \widetilde{s}_{j}^{\left(g_{j}\right)}\right)+\frac{n}{2}-1\right) .
$$


2.2. Prioritized Aggregation Operators. The prioritized average (PA) operator is an aggregation operator in which there exists a prioritization relationship between the arguments [36]. The prioritizations of arguments are modeled by using importance weights in which the weights associated with the lower priority criteria are related to the satisfaction of the higher priority criteria, which were defined as follows.

Definition 4 (see [37]). Let $C=\left\{C_{1}, C_{2}, \ldots, C_{n}\right\}$ be a collection of the criteria and that there is a prioritization between the criteria expressed by the linear ordering $C_{1}>$ $C_{2}>\cdots>C_{n}$, indicating that criteria $C_{j}$ have a higher priority than $C_{k}$, if $1 \leq j<k \leq n$. The value $C_{j}(x)$ is the performance of any alternative $x$ under attribute $C_{j}$ and satisfies $C_{j}(x) \in[0,1]$. If

$$
\operatorname{PWA}\left(C_{j}(x)\right)=\sum_{j=1}^{n} \omega_{j} C_{j}(x)
$$

where $\omega_{j}=T_{j} / \sum_{j=1}^{n} T_{j}, T_{j}=\prod_{k=1}^{j-1} C_{k}(x)(j=2, \ldots, n), T_{1}=$ 1 , then PWA is called the prioritized weighted average (PWA) operator.

The PWA operator emphasizes the situation where lack of satisfaction of higher priority criteria cannot be compensated for by satisfaction of lower priority criteria. The PWA operator is monotonic, bounded, and idempotent [37].

Afterwards, Yager embedded priority weights into the importance weighted OWA operator and presented a prioritized ordered weighted average (POWA) operator.

Definition 5 (see [43]). An OWA operator of dimension $n$ is a mapping OWA : $R^{n} \rightarrow R$ so that

$$
\operatorname{OWA}\left(a_{1}, a_{2}, \ldots, a_{n}\right)=\sum_{j=1}^{n} w_{j} a_{\sigma(j)}
$$

where $\sigma(\cdot): 1,2, \ldots, n \rightarrow 1,2, \ldots, n$ is a permutation function such that $\sigma(j-1) \geq \sigma(j), w_{j}(j=2, \ldots, n)$ are ordered weights such that

$$
w_{j}=Q\left(\frac{j}{n}\right)-Q\left(\frac{j-1}{n}\right), \quad j=1,2, \ldots, n
$$

in which $Q:[0,1] \rightarrow[0,1]$ is a basic unit-interval monotonic (BUM) function having the following properties: (1) $Q(0)=0$, (2) $Q(1)=1$, (3) $Q(x) \geq Q(y)$, if $x \geq y$.

In [44], Yager considered the situation that the argument variable $a_{j}$ is associated with an importance $v_{j}$ and gave a policy to derive ordered weights of OWA operator based on the importance of argument variable:

$$
w_{j}=Q\left(\frac{\sum_{k=1}^{j} v_{\sigma(k)}}{\sum_{k=1}^{n} v_{\sigma(k)}}\right)-Q\left(\frac{\sum_{k=1}^{j-1} v_{\sigma(k)}}{\sum_{k=1}^{n} v_{\sigma(k)}}\right), \quad j=1,2, \ldots, n .
$$

Substituting (9) into (7), we get

$$
\begin{aligned}
\operatorname{OWA} & \left(\left(a_{1}, v_{1}\right),\left(a_{2}, v_{2}\right), \ldots,\left(a_{n}, v_{n}\right)\right) \\
& =\sum_{j=1}^{n}\left(Q\left(\frac{\sum_{k=1}^{j} v_{\sigma(k)}}{\sum_{k=1}^{n} v_{\sigma(k)}}\right)-Q\left(\frac{\sum_{k=1}^{j-1} v_{\sigma(k)}}{\sum_{k=1}^{n} v_{\sigma(k)}}\right)\right) a_{\sigma(j)} .
\end{aligned}
$$

According to the importance weighted OWA operator, Yager takes the prioritized weights as a special importance weights and normally presented the prioritized OWA operator [38], which was defined as follows.

Definition 6. Let $C=\left\{C_{1}, C_{2}, \ldots, C_{n}\right\}$ be a collection of criteria and that there is a prioritization between the criteria expressed by the linear ordering $C_{1}>C_{2}>\cdots>C_{n}$, indicating attribute $C_{j}$ has a higher priority than $C_{k}$, if $1 \leq j<$ $k \leq n$. The value $C_{j}(x)$ is the performance of any alternative $x$ under attribute $C_{j}$ and satisfies $C_{j}(x) \in[0,1]$. If

$$
\begin{gathered}
\operatorname{POWA}\left(C_{j}(x)\right)=\sum_{j=1}^{n} w_{j} C_{\sigma(j)}(x) \\
w_{j}=Q\left(\frac{\sum_{k=1}^{j} T_{\sigma(k)}}{\sum_{j=1}^{n} T_{j}}\right)-Q\left(\frac{\sum_{k=1}^{j-1} T_{\sigma(k)}}{\sum_{j=1}^{n} T_{j}}\right), \quad j=1,2, \ldots, n,
\end{gathered}
$$

where $\sigma(\cdot): 1,2, \ldots, n \rightarrow 1,2, \ldots, n$ is a permutation function such that $\sigma(j-1) \geq \sigma(j), T_{j}=\prod_{k=1}^{j-1} C_{k}(x)(j=$ $2, \ldots, n), T_{1}=1, T_{\sigma(0)}=0$. Then POWA is called the prioritized ordered weighting average (POWA) operator.

The POWA operator is also monotonic, bounded, and idempotent.

\section{Multigranular Uncertain Linguistic Prioritized Operators}

In this section, we will investigate the prioritized aggregation operators under multigranular uncertain linguistic environments.

3.1. Multigranular Uncertain Linguistic Prioritized WA Operator. Based on Definition 4 and the operational laws of multigranular uncertain linguistic variables, we give the definition of the multigranular uncertain linguistic prioritized weighted average (MULPWA) operator as follows.

Definition 7. Let $\widetilde{s}_{j}^{\left(g_{j}\right)}=\left[s_{\alpha_{j}}^{\left(g_{j}\right)}, s_{\beta_{j}}^{\left(g_{j}\right)}\right], s_{\alpha_{j}}^{\left(g_{j}\right)}, s_{\beta_{j}}^{\left(g_{j}\right)} \in \bar{S}^{\left(g_{j}\right)}(j=$ $1,2, \ldots, n)$ be a collection of multi- granular uncertain linguistic variables (MULV), which are prioritized such that 
$\widetilde{s}_{i}^{\left(g_{i}\right)}>\widetilde{s}_{j}^{\left(g_{j}\right)}$ if $i<j$, and then the MULPWA operator is defined as follows:

$$
\begin{aligned}
& \operatorname{MULPWA}\left(\widetilde{s}_{1}^{\left(g_{1}\right)}, \widetilde{s}_{2}^{\left(g_{2}\right)}, \ldots, \widetilde{s}_{n}^{\left(g_{n}\right)}\right) \\
& =\frac{T_{1}}{\sum_{j=1}^{n} T_{j}} \widetilde{s}_{1}^{\left(g_{1}\right)} \oplus \frac{T_{2}}{\sum_{j=1}^{n} T_{j}} \widetilde{s}_{2}^{\left(g_{2}\right)} \oplus \cdots \oplus \frac{T_{n}}{\sum_{j=1}^{n} T_{j}} \widetilde{s}_{n}^{\left(g_{n}\right)} \\
& =\bigoplus_{j=1}^{n}\left(\frac{T_{j} \widetilde{s}_{j}^{\left(g_{j}\right)}}{\sum_{j=1}^{n} T_{j}}\right),
\end{aligned}
$$

where $T_{j}=\prod_{k=1}^{j-1} P\left(\widetilde{s}_{k}^{\left(g_{k}\right)}\right)(j=2, \ldots, n), T_{1}=1$, and $P\left(\widetilde{s}_{k}^{\left(g_{k}\right)}\right)$ is the possibility degree of $\widetilde{s}_{k}^{\left(g_{k}\right)}$ among the $\widetilde{s}_{j}^{\left(g_{j}\right)}(j=$ $1,2, \ldots, n)$. In particular, if $g_{1}=g_{2}=\cdots=g_{n}$, then the MULPWA operator returns to an uncertain linguistic prioritized weighted average (ULPWA) operator; if $\widetilde{s}_{\alpha_{j}}\left(g_{j}\right)=$ $\widetilde{s}_{\beta_{j}}^{\left(g_{j}\right)}$ for all $j=1,2, \ldots, n$, then the MULPWA operator returns to a multigranular linguistic prioritized weighted average (MLPWA) operator.

From (12), we know that:

(1) since $T_{j} \geq T_{k}$ for $j<k$, then a criterion can never have a bigger priority weight than a criterion that has a higher priority than it;

(2) the MULPWA operator consists of the following three steps:

(a) calculate the relative possibility degrees $P\left(\widetilde{s}_{j}\left(g_{j}\right)\right)(j=1,2, \ldots, n)$ of the multigranular uncertain linguistic arguments variables $\widetilde{s}_{j}^{\left(g_{j}\right)}(j=1,2, \ldots, n)$ by (3) and (5);

(b) determine the prioritized levels $T_{j}=$ $\prod_{k=1}^{j-1} P\left(\widetilde{s}_{k}^{\left(g_{k}\right)}\right)(j=2, \ldots, n)$ and $\sum_{j=1}^{n} T_{j} ;$

(c) derive the aggregated results $\widehat{s}_{j}^{\left(g_{j}\right)}(j=$ $1,2, \ldots, n)$ according to $\oplus_{j=1}^{n}\left(T_{j} / \sum_{j=1}^{n} T_{j}\right) \widetilde{s}_{j}^{(}\left(g_{j}\right)$.

Example 8. Given the collection of multigranular uncertain linguistic variables $\widehat{s}_{1}^{(7)}=\left[s_{3}^{(7)}, s_{5}^{(7)}\right], \widetilde{s}_{2}^{(11)}=\left[s_{4}^{(11)}, s_{6}^{(11)}\right], \widetilde{s}_{3}^{(5)}=$ $\left[s_{2}^{(5)}, s_{3}^{(5)}\right]$, and $\widetilde{s}_{4}^{(9)}=\left[s_{5}^{(9)}, s_{6}^{(9)}\right]$, the prioritized relations are $x_{1}>x_{2}>x_{3}>x_{4}$.

By (3), we have

$$
\begin{aligned}
P\left(\widetilde{s}_{1}^{(7)} \geq\right. & \left.\widetilde{s}_{2}^{(11)}\right) \\
= & (\max \{0,(11-1) 5-(7-1) 4\} \\
& \quad-\max \{0,(11-1) 3-(7-1) 6\}) \\
& \times((11-1)(5-3)+(7-1)(6-4))^{-1}=0.8125 .
\end{aligned}
$$

TABLE 1: Possibility degree matrix.

\begin{tabular}{lcccc}
\hline & $\widetilde{s}_{1}^{(7)}$ & $\widetilde{s}_{2}^{(11)}$ & $\widetilde{s}_{3}^{(5)}$ & $\widetilde{s}_{4}^{(9)}$ \\
\hline$\widetilde{s}_{1}^{(7)}$ & 0.5000 & 0.8125 & 0.5714 & 0.4545 \\
$\widetilde{s}_{2}^{(11)}$ & 0.1875 & 0.5000 & 0.2222 & 0.0000 \\
$\widetilde{s}_{3}^{(5)}$ & 0.4268 & 0.7778 & 0.5000 & 0.3333 \\
$\widetilde{s}_{4}^{(9)}$ & 0.5455 & 1.0000 & 0.6667 & 0.5000 \\
\hline
\end{tabular}

Similarly, we can derive all pair comparisons among the multigranular uncertain linguistic values and form a possibility degree matrix as shown in Table 1 .

Utilize (5) to derive the relative possibility degrees $P\left(\widetilde{s}_{i}^{\left(g_{i}\right)}\right)(i=1,2,3,4)$ over all the other alternatives:

$$
\begin{aligned}
P\left(\widetilde{s}_{1}^{(7)}\right)= & \frac{1}{4(4-1)} \\
& \times(0.5000+0.8125+0.5714+0.4545+2-1) \\
= & 0.2782 \\
P\left(\widetilde{s}_{2}^{(11)}\right)= & \frac{1}{4(4-1)} \\
& \times(0.1875+0.5000+0.2222+0.0000+1) \\
= & 0.1592 \\
P\left(\widetilde{s}_{3}^{(5)}\right)= & \frac{1}{4(4-1)} \\
& \times(0.4268+0.7778+0.5000+0.3333+1) \\
= & 0.2532 \\
P\left(\widetilde{s}_{4}^{(9)}\right)= & \frac{1}{4(4-1)} \\
& \times(0.5455+1.0000+0.6667+0.5000+1) \\
= & 0.3094 .
\end{aligned}
$$

Then determine the prioritized levels $T_{j}(j=$ $1,2,3,4) T_{1}=1, T_{2}=0.2782, T_{3}=0.2782 \times 0.1592=$ $0.0443, T_{4}=0.2782 \times 0.1592 \times 0.2523=0.0112$, and $\sum_{j=1}^{4} T_{j}=1.3337$.

Thus,

$$
\begin{aligned}
\operatorname{MULPWA}\left(\widetilde{s}_{1}^{(7)}, \widetilde{s}_{2}^{(11)}, \widetilde{s}_{3}^{(5)}, \widetilde{s}_{4}^{(9)}\right) \\
=\frac{1}{1.3337}\left[s_{3}^{(7)}, s_{5}^{(7)}\right] \oplus \frac{0.2782}{1.3337}\left[s_{4}^{(11)}, s_{6}^{(11)}\right] \\
\oplus \frac{0.0443}{1.3337}\left[s_{2}^{(5)}, s_{3}^{(5)}\right] \oplus \frac{0.0112}{1.3337}\left[s_{5}^{(9)}, s_{6}^{(9)}\right] \\
=\frac{1}{1.3337}\left[s_{(11-1) /(7-1) \times 3}^{(11)}, s_{(11-1) /(7-1) \times 5}^{(11)}\right] \\
\oplus \frac{0.2782}{1.3337}\left[s_{(11-1) /(11-1) \times 4}^{(11)}, s_{(11-1) /(11-1) \times 6}^{(11)}\right]
\end{aligned}
$$




$$
\begin{aligned}
& \oplus \frac{0.0443}{1.3337}\left[s_{(11-1) /(5-1) \times 2}^{(11)}, s_{(11-1) /(5-1) \times 3}^{(11)}\right] \\
& \oplus \frac{0.0112}{1.3337}\left[s_{(11-1) /(9-1) \times 5}^{(11)}, s_{(11-1) /(9-1) \times 6}^{(11)}\right] \\
= & {\left[s_{4.802}^{(11)}, s_{7.8121}^{(11)}\right] . }
\end{aligned}
$$

Similar to the PWA operator, it can be easily proved that the MULPWA operator has the following properties.

Theorem 9 (idempotency). Let $\widetilde{s}_{j}^{\left(g_{j}\right)}=\left[s_{\alpha_{j}}^{\left(g_{j}\right)}, s_{\beta_{j}}^{\left(g_{j}\right)}\right]$, $s_{\alpha_{j}}^{\left(g_{j}\right)}, s_{\beta_{j}}^{\left(g_{j}\right)} \in \bar{S}^{\left(g_{j}\right)}(j=1,2, \ldots, n)$ be a collection of $M U L V$, where $T_{j}=\prod_{k=1}^{j-1} P\left(\widetilde{s}_{k}^{\left(g_{k}\right)}\right)(j=2, \ldots, n), T_{1}=1$, and $P\left(\widetilde{s}_{k}^{\left(g_{k}\right)}\right)$ is the possibility degree of $\widetilde{s}_{k}^{\left(g_{k}\right)}$. If $\widetilde{s}_{j}^{\left(g_{j}\right)}=\widetilde{s}^{\prime}$ for all $j=1,2, \ldots, n$, then

$$
\operatorname{MULPWA}\left(\widetilde{s}_{1}^{\left(g_{1}\right)}, \widetilde{s}_{2}^{\left(g_{2}\right)}, \ldots, \widetilde{s}_{n}^{\left(g_{n}\right)}\right)=\widetilde{s}^{\prime}
$$

Proof. Since $\widetilde{s}_{j}\left(g_{j}\right)=\vec{s}$ for all $j=1,2, \ldots, n$, we can get $P\left(\widetilde{s}_{j}\left(g_{j}\right)\right)=0.5$ for all $j=1,2, \ldots, n$, then $T_{1}^{\prime}=1, T_{j}^{\prime}=$ $(0.5)^{j-1}(j=2, \ldots, n)$.

$$
\begin{aligned}
& \operatorname{MUlpwA}\left(\widetilde{s}_{1}^{\left(g_{1}\right)}, \widetilde{s}_{2}^{\left(g_{2}\right)}, \ldots, \widetilde{s}_{n}^{\left(g_{n}\right)}\right) \\
& =\bigoplus_{j=1}^{n}\left(\frac{T_{j} \widetilde{s}_{j}^{\left(g_{j}\right)}}{\sum_{j=1}^{n} T_{j}}\right)=\bigoplus_{j=1}^{n}\left(\frac{T_{j}^{\prime} \vec{s}^{\prime}}{\sum_{j=1}^{n} T_{j}^{\prime}}\right)=\vec{s}^{\prime} .
\end{aligned}
$$

Theorem 10 (boundedness). Let $\widetilde{s}_{j}^{\left(g_{j}\right)}=\left[s_{\alpha_{j}}^{\left(g_{j}\right)}, s_{\beta_{j}}^{\left(g_{j}\right)}\right]$, $s_{\alpha_{j}}^{\left(g_{j}\right)}, s_{\beta_{j}}^{\left(g_{j}\right)} \in \bar{S}^{\left(g_{j}\right)}(j=1,2, \ldots, n)$ be a collection of $M U L V$, $T_{j}=\prod_{k=1}^{j-1} P\left(\widetilde{s}_{k}^{\left(g_{k}\right)}\right)(j=2, \ldots, n), T_{1}=1$, and $P\left(\widetilde{s}_{k}^{\left(g_{k}\right)}\right)$ is the possibility degree of $\widetilde{s}_{k}^{\left(g_{k}\right)}$, and then

$$
\begin{aligned}
\min _{j}\left\{\widetilde{s}_{j}^{\left(g_{j}\right)}\right\} & \leq \operatorname{MULPWA}\left(\widetilde{s}_{1}^{\left(g_{1}\right)}, \widetilde{s}_{2}^{\left(g_{2}\right)}, \ldots, \widetilde{s}_{n}^{\left(g_{n}\right)}\right) \\
& \leq \max _{j}\left\{\widetilde{s}_{j}^{\left(g_{j}\right)}\right\} .
\end{aligned}
$$

Proof. Since

$$
\begin{aligned}
\operatorname{MULPWA} & \left(\widetilde{s}_{1}^{\left(g_{1}\right)}, \widetilde{s}_{2}^{\left(g_{2}\right)}, \ldots, \widetilde{s}_{n}^{\left(g_{n}\right)}\right) \\
= & \bigoplus_{j=1}^{n}\left(\frac{T_{j} \widetilde{s}_{j}^{\left(g_{j}\right)}}{\sum_{j=1}^{n} T_{j}}\right) \leq \bigoplus_{j=1}^{n}\left(\frac{T_{j} \widetilde{s}_{j}^{*\left(g_{j}\right)}}{\sum_{j=1}^{n} T_{j}}\right) \\
= & \bigoplus_{j=1}^{n}\left(\frac{T_{j}^{\prime} \widetilde{s}_{j}^{*\left(g_{j}\right)}}{\sum_{j=1}^{n} T l_{j}}\right) \\
= & \widetilde{s}_{j}^{*\left(g_{j}\right)}=\max _{j}\left\{\widetilde{s}_{j}^{\left(g_{j}\right)}\right\} \operatorname{MULPWA}\left(\widetilde{s}_{1}^{\left(g_{1}\right)}, \widetilde{s}_{2}^{\left(g_{2}\right)}, \ldots, \widetilde{s}_{n}^{\left(g_{n}\right)}\right)
\end{aligned}
$$

$$
\begin{aligned}
& =\bigoplus_{j=1}^{n}\left(\frac{T_{j} \widetilde{s}_{j}^{\left(g_{j}\right)}}{\sum_{j=1}^{n} T_{j}}\right) \geq \bigoplus_{j=1}^{n}\left(\frac{T_{j} \widetilde{s}_{j}^{-\left(g_{j}\right)}}{\sum_{j=1}^{n} T_{j}}\right) \\
& =\bigoplus_{j=1}^{n}\left(\frac{T_{j}^{\prime} \widetilde{s}_{j}^{-\left(g_{j}\right)}}{\sum_{j=1}^{n} T_{j}^{\prime}}\right) \\
& =\widetilde{s}_{j}^{-\left(g_{j}\right)}=\min _{j}\left\{\widetilde{s}_{j}^{\left(g_{j}\right)}\right\},
\end{aligned}
$$

thus $\min _{j}\left\{\widetilde{s}_{j}^{\left(g_{j}\right)}\right\} \leq \operatorname{MULPWA}\left(\widetilde{s}_{1}^{\left(g_{1}\right)}, \widetilde{s}_{2}^{\left(g_{2}\right)}, \ldots, \widetilde{s}_{n}^{\left(g_{n}\right)}\right) \leq$ $\max _{j}\left\{\widetilde{s}_{j}\left(g_{j}\right)\right\}$.

Theorem 11 (monotonicity). Let $\widetilde{s}_{j}^{\left(g_{j}\right)}=\left[s_{\alpha_{j}}^{\left(g_{j}\right)}, s_{\beta_{j}}^{\left(g_{j}\right)}\right]$, $s_{\alpha_{j}}^{\left(g_{j}\right)}, s_{\beta_{j}}^{\left(g_{j}\right)} \in \bar{S}^{\left(g_{j}\right)}, \widetilde{s}_{j}^{\prime\left(g_{j}\right)}=\left[s_{\alpha_{j}}^{\prime\left(g_{j}\right)}, s_{\beta_{j}}^{\prime\left(g_{j}\right)}\right], s_{\alpha_{j}}^{\prime\left(g_{j}\right)}, s_{\beta_{j}}^{\prime\left(g_{j}\right)} \in \bar{S}^{\prime\left(g_{j}\right)}$ $(j=1,2, \ldots, n)$ be two collections of MULV, where $T_{j}=\prod_{k=1}^{j-1} P\left(\widetilde{s}_{k}^{\left(g_{k}\right)}\right), T_{j}^{\prime}=\prod_{k=1}^{j-1} P\left(\widetilde{s}_{k}^{\left(g_{k}\right)}\right)(j=2, \ldots, n)$, $T_{1}=1, T_{1}^{\prime}=1$, and $P\left(\widetilde{s}_{k}^{\left(g_{k}\right)}\right)$ and $P\left(\widetilde{s}_{k}^{\left(g_{k}\right)}\right)$ are the possibility degrees of $\widetilde{s}_{k}^{\left(g_{k}\right)}$ and $\vec{s}_{k}^{\left(g_{k}\right)}$, respectively. If $\widetilde{s}_{j}^{\left(g_{j}\right)} \leq \widetilde{s}_{j}^{\prime\left(g_{j}\right)}$ for all $j=1,2, \ldots, n$, then

$$
\begin{aligned}
& \operatorname{MULPWA}\left(\widetilde{s}_{1}^{\left(g_{1}\right)}, \widetilde{s}_{2}^{\left(g_{2}\right)}, \ldots, \widetilde{s}_{n}^{\left(g_{n}\right)}\right) \\
& \quad \leq \operatorname{MULPWA}\left(\widetilde{s}_{1}^{\left(g_{1}\right)}, \widetilde{s}_{2}^{\prime\left(g_{2}\right)}, \ldots, \widetilde{s}_{n}^{\left(g_{n}\right)}\right) .
\end{aligned}
$$

Proof. Since $\operatorname{MULPWA}\left(\widetilde{s}_{1}^{\left(g_{1}\right)}, \widetilde{s}_{2}^{\left(g_{2}\right)}, \ldots, \widetilde{s}_{n}^{\left(g_{n}\right)}\right)=\oplus_{j=1}^{n}$ $\left(T_{j} \widetilde{s}_{j}^{\left(g_{j}\right)} / \sum_{j=1}^{n} T_{j}\right)$, and $\operatorname{MULPWA}\left(\widetilde{s}_{1}^{\left(g_{1}\right)}, \widetilde{s}_{2}^{\left(g_{2}\right)}, \ldots, \widetilde{s}_{n}^{\left(g_{n}\right)}\right)$ $=\oplus_{j=1}^{n}\left(T_{j}^{\prime} \widetilde{s}_{j}^{\prime\left(g_{j}\right)} / \sum_{j=1}^{n} T_{j}^{\prime}\right)$ and meanwhile, since $\widetilde{s}_{j}^{\left(g_{j}\right)} \leq \widetilde{s}_{j}^{\prime\left(g_{j}\right)}$ for all $j=1,2, \ldots, n, T_{j} \geq T_{j}^{\prime}, T_{1} \geq T_{2} \geq \cdots \geq T_{n}$, and $T_{1}^{\prime} \geq T_{2}^{\prime} \geq \cdots \geq T_{n}^{\prime}$.

Thus, $\operatorname{MULPWA}\left(\widetilde{s}_{1}^{\left(g_{1}\right)}, \widetilde{s}_{2}^{\left(g_{2}\right)}, \ldots, \widetilde{s}_{n}^{\left(g_{n}\right)}\right) \leq \operatorname{MULPWA}\left(\widetilde{s}_{1}^{\left(g_{1}\right)}\right.$, $\left.\widetilde{s}_{2}^{\left(g_{2}\right)}, \ldots, \widetilde{s}_{n}^{\left(g_{n}\right)}\right)$.

Theorem 12. Let $\widetilde{s}_{j}^{\left(g_{j}\right)}=\left[s_{\alpha_{j}}^{\left(g_{j}\right)}, s_{\beta_{j}}^{\left(g_{j}\right)}\right], s_{\alpha_{j}}^{\left(g_{j}\right)}, s_{\beta_{j}}^{\left(g_{j}\right)} \in \bar{S}^{\left(g_{j}\right)}(j=1$, $2, \ldots, n)$ be a collection of MULV, where $T_{j}=\prod_{k=1}^{j-1}$ $P\left(\widetilde{s}_{k}^{\left(g_{k}\right)}\right)(j=2, \ldots, n), T_{1}=1$, and $P\left(\widetilde{s}_{k}^{\left(g_{k}\right)}\right)$ is the possibility degree of $\widetilde{s}_{k}^{\left(g_{k}\right)}$. If $\mu>0$, then

$$
\begin{aligned}
& \operatorname{MULPWA}\left(\mu \widetilde{s}_{1}^{\left(g_{1}\right)}, \mu \widetilde{s}_{2}^{\left(g_{2}\right)}, \ldots, \mu \widetilde{s}_{n}^{\left(g_{n}\right)}\right) \\
& =\mu \operatorname{MULPWA}\left(\widetilde{s}_{1}^{\left(g_{1}\right)}, \widetilde{s}_{2}^{\left(g_{2}\right)}, \ldots, \widetilde{s}_{n}^{\left(g_{n}\right)}\right) .
\end{aligned}
$$

Proof. Since

$$
\begin{aligned}
P\left(\mu \widetilde{s}_{1}^{\left(g_{1}\right)} \geq \mu \widetilde{s}_{2}^{\left(g_{2}\right)}\right) & \\
= & \left(\max \left\{0,\left(g_{2}-1\right) \mu \beta_{1}-\left(g_{1}-1\right) \mu \alpha_{2}\right\}\right. \\
& \left.\quad-\max \left\{0,\left(g_{2}-1\right) \mu \alpha_{1}-\left(g_{1}-1\right) \mu \beta_{2}\right\}\right) \\
& \times\left(\left(g_{2}-1\right)\left(\mu \beta_{1}-\mu \alpha_{1}\right)+\left(g_{1}-1\right)\left(\mu \beta_{2}-\mu \alpha_{2}\right)\right)^{-1}
\end{aligned}
$$




$$
\begin{aligned}
= & \left(\max \left\{0,\left(g_{2}-1\right) \beta_{1}-\left(g_{1}-1\right) \alpha_{2}\right\}\right. \\
& \left.-\max \left\{0,\left(g_{2}-1\right) \alpha_{1}-\left(g_{1}-1\right) \beta_{2}\right\}\right) \\
& \times\left(\left(g_{2}-1\right)\left(\beta_{1}-\alpha_{1}\right)+\left(g_{1}-1\right)\left(\beta_{2}-\alpha_{2}\right)\right)^{-1} \\
= & P\left(\widetilde{s}_{1}^{\left(g_{1}\right)} \geq \widetilde{s}_{2}^{\left(g_{2}\right)}\right),
\end{aligned}
$$

then $P\left(\mu \widetilde{s}_{1}^{\left(g_{1}\right)}\right)=P\left(\widetilde{s}_{1}^{\left(g_{1}\right)}\right), g_{1}=g_{2}=\cdots=g_{n}$, and then

$$
\begin{aligned}
& \operatorname{MUPWG}\left(\mu \widetilde{s}_{1}^{\left(g_{1}\right)}, \mu \widetilde{s}_{2}^{\left(g_{2}\right)}, \ldots, \mu \widetilde{s}_{n}^{\left(g_{n}\right)}\right) \\
& =\mu \operatorname{MUPWG}\left(\vec{s}_{1}^{\left(g_{1}\right)}, \widetilde{s_{2}}\left(g_{2}\right), \ldots, \widetilde{s}_{n}^{\left(g_{n}\right)}\right) .
\end{aligned}
$$

3.2. Multigranular Uncertain Linguistic Prioritized OWA Operator. Based on Definition 6 and the operational laws of multigranular uncertain linguistic variables, we give the definition of the multigranular uncertain linguistic prioritized ordered weighted average (MULPOWA) operator as follows.

Definition 13. Let $\widetilde{s}_{j}^{\left(g_{j}\right)}=\left[s_{\alpha_{j}}^{\left(g_{j}\right)}, s_{\beta_{j}}^{\left(g_{j}\right)}\right], s_{\alpha_{j}}^{\left(g_{j}\right)}, s_{\beta_{j}}^{\left(g_{j}\right)} \in \bar{S}^{\left(g_{j}\right)}(j=$ $1,2, \ldots, n)$ be a collection of multigranular uncertain linguistic variables (MULV), which are prioritized such that $\widetilde{s}_{i}^{\left(g_{i}\right)}>$ $\widetilde{s}_{j}\left(g_{j}\right)$. If $i<j$, then the MULPOWA operator is defined as follows:

$$
\begin{aligned}
& \operatorname{MULPOWA}\left(\widetilde{s}_{1}^{\left(g_{1}\right)}, \widetilde{s}_{2}^{\left(g_{2}\right)}, \ldots, \widetilde{s}_{n}^{\left(g_{n}\right)}\right) \\
& =\bigoplus_{j=1}^{n}\left(Q\left(\frac{\sum_{k=1}^{j} T_{\sigma(k)}}{\sum_{j=1}^{n} T_{j}}\right)-Q\left(\frac{\sum_{k=1}^{j-1} T_{\sigma(k)}}{\sum_{j=1}^{n} T_{j}}\right)\right) \widetilde{s}_{\sigma(j)}\left(g_{j}\right),
\end{aligned}
$$

where $\sigma(\cdot): 1,2, \ldots, n \rightarrow 1,2, \ldots, n$ is a permutation function such that $\sigma(j-1) \geq \sigma(j), T_{j}=\prod_{k=1}^{j-1} P\left(\widetilde{s}_{k}^{\left(g_{k}\right)}\right)(j=$ $2, \ldots, n), T_{1}=1, T_{\sigma(0)}=0$, and $P\left(\widetilde{s}_{k}\left(g_{k}\right)\right)$ is the possibility degree of $\widetilde{s}_{k}^{\left(g_{k}\right)}$. In particular, if $g_{1}=g_{2}=\cdots=g_{n}$, then the MULPOWA operator returns to an uncertain linguistic prioritized ordered weighted average (ULPOWA) operator; if $\widetilde{s}_{\alpha_{j}}\left(g_{j}\right)=\widetilde{s}_{\beta_{j}}\left(g_{j}\right)$ for all $j=1,2, \ldots, n$, then the MULPOWA operator returns to a multigranular linguistic prioritized weighted average (MLPWA) operator.

The aggregation process of the MULPOWA operator consists of the following four steps:

(1) calculate the possibility degrees $P\left(\widetilde{s}_{j}^{\left(g_{j}\right)}\right)(j=$ $1,2, \ldots, n)$ of the multigranular; uncertain linguistic arguments variables $\widetilde{s}_{j}\left(g_{j}\right) \quad(j=1,2, \ldots, n)$ by (3) and (5);

(2) determine the prioritized levels $T_{j}=$ $\prod_{k=1}^{j-1} P\left(\widetilde{s}_{k}^{\left(g_{k}\right)}\right)(j=2, \ldots, n)$ and $\sum_{j=1}^{n} T_{j} ;$
(3) reorder the input arguments $a_{j}$ in descending order and associate with $T_{j}$;

(4) derive the aggregated results of $\widetilde{s}_{j}\left(g_{j}\right) \quad(j=1,2, \ldots, n)$ according to

$$
\bigoplus_{j=1}^{n}\left(Q\left(\frac{\sum_{k=1}^{j} T_{\sigma(k)}}{\sum_{j=1}^{n} T_{j}}\right)-Q\left(\frac{\sum_{k=1}^{j-1} T_{\sigma(k)}}{\sum_{j=1}^{n} T_{j}}\right)\right) \widetilde{s}_{\sigma(j)}^{\left(g_{j}\right)} .
$$

Example 14. Given the collection of multigranular uncertain linguistic variables $\widehat{s}_{1}^{(7)}=\left[s_{3}^{(7)}, s_{5}^{(7)}\right], \widetilde{s}_{2}^{(11)}=\left[s_{4}^{(11)}, s_{6}^{(11)}\right], \widetilde{s}_{3}^{(5)}=$ $\left[s_{2}^{(5)}, s_{3}^{(5)}\right]$, and $\widetilde{s}_{4}^{(9)}=\left[s_{5}^{(9)}, s_{6}^{(9)}\right]$, the prioritized relations are $x_{1}>x_{2}>x_{3}>x_{4}$.

Since $P\left(\widetilde{s}_{4}^{(9)}\right)=0.3094>P\left(\widetilde{s}_{1}^{(7)}\right)=0.2782>P\left(\widetilde{s}_{3}^{(5)}\right)=$ $0.2532>P\left(\widetilde{s}_{2}^{(11)}\right)=0.1592, \widetilde{s}_{4}^{(9)}>\widetilde{s}_{1}^{(7)}>\widetilde{s}_{3}^{(5)}>\widetilde{s}_{2}^{(11)} \cdot T_{1}=1$, $T_{2}=0.2782, T_{3}=0.0443, T_{4}=0.0112$, and $\sum_{j=1}^{4} T_{j}=1.3337$, $Q(r)=r^{2}$. Consider

$$
\begin{aligned}
& \operatorname{MULPOWA}\left(\widetilde{s}_{1}^{(7)}, \widetilde{s}_{2}^{(11)}, \widetilde{s}_{3}^{(5)}, \widetilde{s}_{4}^{(9)}\right) \\
& =\left(Q\left(\frac{T_{4}}{\sum_{j=1}^{4} T_{j}}\right)-Q\left(\frac{T_{0}}{\sum_{j=1}^{4} T_{j}}\right)\right) \widetilde{s}_{4}^{(9)} \\
& \oplus\left(Q\left(\frac{T_{4}+T_{1}}{\sum_{j=1}^{4} T_{j}}\right)-Q\left(\frac{T_{4}}{\sum_{j=1}^{4} T_{j}}\right)\right) \widetilde{s}_{1}^{(7)} \\
& \oplus\left(Q\left(\frac{T_{4}+T_{1}+T_{3}}{\sum_{j=1}^{4} T_{j}}\right)-Q\left(\frac{T_{4}+T_{1}}{\sum_{j=1}^{4} T_{j}}\right)\right) \widetilde{s}_{3}^{(5)} \\
& \oplus\left(Q\left(\frac{T_{4}+T_{1}+T_{3}+T_{2}}{\sum_{j=1}^{4} T_{j}}\right)\right. \\
& \left.-Q\left(\frac{T_{4}+T_{1}+T_{3}}{\sum_{j=1}^{4} T_{j}}\right)\right) \widetilde{s}_{2}^{(11)} \\
& =(Q(0.0084)-Q(0))\left[s_{5}^{(9)}, s_{6}^{(9)}\right] \\
& \oplus(Q(0.7582)-Q(0.0084))\left[s_{3}^{(7)}, s_{5}^{(7)}\right] \\
& \oplus(Q(0.7914)-Q(0.7582))\left[s_{2}^{(5)}, s_{3}^{(5)}\right] \\
& \oplus(Q(1)-Q(0.7914))\left[s_{4}^{(11)}, s_{6}^{(11)}\right] \\
& =0.0001\left[s_{(11-1) /(9-1) \times 5}^{(11)}, s_{(11-1) /(9-1) \times 6}^{(11)}\right] \\
& +0.5748\left[s_{(11-1) /(7-1) \times 3}^{(11)}, s_{(11-1) /(7-1) \times 5}^{(11)}\right] \\
& +0.0514\left[s_{(11-1) /(5-1) \times 2}^{(11)}, s_{(11-1) /(5-1) \times 3}^{(11)}\right] \\
& +0.3737\left[s_{(11-1) /(11-1) \times 4}^{(11)}, s_{(11-1) /(11-1) \times 6}^{(11)}\right] \\
& =\left[s_{4.4}^{(11)}, s_{7.07}^{(11)}\right] \text {. }
\end{aligned}
$$


Similarly to Theorems $9-12$ and (24), it can be easily proved that the MULPOWA operator has the following properties.

Theorem 15 (idempotency). Let $\widetilde{s}_{j}^{\left(g_{j}\right)}=\left[s_{\alpha_{j}}^{\left(g_{j}\right)}, s_{\beta_{j}}^{\left(g_{j}\right)}\right], s_{\alpha_{j}}^{\left(g_{j}\right)}$, $s_{\beta_{j}}^{\left(g_{j}\right)} \in \bar{S}^{\left(g_{j}\right)}(j=1,2, \ldots, n)$ be a collection of MULV, where $T_{j}=\prod_{k=1}^{j-1} P\left(\widetilde{s}_{k}^{\left(g_{k}\right)}\right)(j=2, \ldots, n), T_{1}=1, T_{\sigma(0)}=0$, and $P\left(\widetilde{s}_{k}^{\left(g_{k}\right)}\right)$ is the possibility degree of $\widetilde{s}_{k}^{\left(g_{k}\right)}$. If $\widetilde{s}_{j}^{\left(g_{j}\right)}=\vec{s}^{\prime}$ for all $j=1,2, \ldots, n$, then

$$
\operatorname{MULPOWA}\left(\widetilde{s}_{1}^{\left(g_{1}\right)}, \widetilde{s}_{2}^{\left(g_{2}\right)}, \ldots, \widetilde{s}_{n}^{\left(g_{n}\right)}\right)=\vec{s}
$$

Theorem 16 (boundedness). Let $\widetilde{s}_{j}^{\left(g_{j}\right)}=\left[s_{\alpha_{j}}^{\left(g_{j}\right)}, s_{\beta_{j}}^{\left(g_{j}\right)}\right], s_{\alpha_{j}}^{\left(g_{j}\right)}$, $s_{\beta_{j}}^{\left(g_{j}\right)} \in \bar{S}^{\left(g_{j}\right)}(j=1,2, \ldots, n)$ be a collection of $M U L V, T_{j}=$ $\prod_{k=1}^{j-1} P\left(\widetilde{s}_{k}^{\left(g_{k}\right)}\right)(j=2, \ldots, n), T_{1}=1, T_{\sigma(0)}=0$, and $P\left(\widetilde{s}_{k}^{\left(g_{k}\right)}\right)$ is the possibility degree of $\widetilde{s}_{k}^{\left(g_{k}\right)}$, and then

$$
\begin{aligned}
\min _{j}\left\{\widetilde{s}_{j}^{\left(g_{j}\right)}\right\} & \leq \operatorname{MULPOWA}\left(\widetilde{s}_{1}^{\left(g_{1}\right)}, \widetilde{s}_{2}^{\left(g_{2}\right)}, \ldots, \widetilde{s}_{n}^{\left(g_{n}\right)}\right) \\
& \leq \max _{j}\left\{\widetilde{s}_{j}^{\left(g_{j}\right)}\right\} .
\end{aligned}
$$

Theorem 17 (monotonicity). Let $\widetilde{s}_{j}^{\left(g_{j}\right)}=\left[s_{\alpha_{j}}^{\left(g_{j}\right)}, s_{\beta_{j}}^{\left(g_{j}\right)}\right], s_{\alpha_{j}}^{\left(g_{j}\right)}$, $s_{\beta_{j}}^{\left(g_{j}\right)} \in \bar{S}^{\left(g_{j}\right)}, \widetilde{s}_{j}^{\left(g_{j}\right)}=\left[s_{\alpha_{j}}^{\prime\left(g_{j}\right)}, s_{\beta_{j}}^{\prime\left(g_{j}\right)}\right], s_{\alpha_{j}}^{\prime\left(g_{j}\right)}, s_{\beta_{j}}^{\prime\left(g_{j}\right)} \in \bar{S}^{\prime\left(g_{j}\right)}$ $(j=1,2, \ldots, n)$ be two collections of $M U L V$, where $T_{j}=$ $\prod_{k=1}^{j-1} P\left(\widetilde{s}_{k}^{\left(g_{k}\right)}\right), T_{j}^{\prime}=\prod_{k=1}^{j-1} P\left(\widetilde{s}_{k}^{\left(g_{k}\right)}\right)(j=2, \ldots, n), T_{1}=1, T_{1}^{\prime}=$ $1, T_{\sigma(0)}=0, T_{\sigma(0)}^{\prime}=0, P\left(\widetilde{s}_{k}^{\left(g_{k}\right)}\right)$ and $P\left(\widetilde{s}_{k}^{\left(g_{k}\right)}\right)$ are the possibility degrees of $\widetilde{s}_{k}^{\left(g_{k}\right)}$ and $\widetilde{s}_{k}^{\left(g_{k}\right)}$, respectively. If $\widetilde{s}_{j}^{\left(g_{j}\right)} \leq \widetilde{s}_{j}^{\prime\left(g_{j}\right)}$ for all $j=1,2, \ldots, n$, then

$$
\begin{aligned}
& \operatorname{MULPOWA}\left(\widetilde{s}_{1}^{\left(g_{1}\right)}, \widetilde{s}_{2}^{\left(g_{2}\right)}, \ldots, \widetilde{s}_{n}^{\left(g_{n}\right)}\right) \\
& \quad \leq \operatorname{MULPOWA}\left(\widetilde{s}_{1}^{\left(g_{1}\right)}, \widetilde{s}_{2}^{\left(g_{2}\right)}, \ldots, \widetilde{s}_{n}^{\left(g_{n}\right)}\right) .
\end{aligned}
$$

Theorem 18. Let $\widetilde{s}_{j}^{\left(g_{j}\right)}=\left[s_{\alpha_{j}}^{\left(g_{j}\right)}, s_{\beta_{j}}^{\left(g_{j}\right)}\right], s_{\alpha_{j}}^{\left(g_{j}\right)}, s_{\beta_{j}}^{\left(g_{j}\right)} \in$ $\bar{S}^{\left(g_{j}\right)}(j=1,2, \ldots, n)$ be a collection of MULV, where $T_{j}=\prod_{k=1}^{j-1} P\left(\widetilde{s}_{k}^{\left(g_{k}\right)}\right)(j=2, \ldots, n), T_{1}=1, T_{\sigma(0)}=0$, and $P\left(\widetilde{s}_{k}^{\left(g_{k}\right)}\right)$ is the possibility degree of $\widetilde{s}_{k}^{\left(g_{k}\right)}$. If $\mu>0$, then

$$
\begin{aligned}
& \operatorname{MULPOWA}\left(\mu \widetilde{s}_{1}^{\left(g_{1}\right)}, \mu \widetilde{s}_{2}^{\left(g_{2}\right)}, \ldots, \mu \widetilde{s}_{n}^{\left(g_{n}\right)}\right) \\
& =\mu \operatorname{MULPOWA}\left(\widetilde{s}_{1}^{\left(g_{1}\right)}, \vec{s}_{2}^{\left(g_{2}\right)}, \ldots, \widetilde{s}_{n}^{\left(g_{n}\right)}\right) .
\end{aligned}
$$

\section{An Approach to Multiple Criteria Group Decision Making with Prioritized Levels}

Let $X=\left\{x_{1}, x_{2}, \ldots, x_{m}\right\}$ be a set of alternatives, $C=$ $\left\{C_{1}, C_{2}, \ldots, C_{n}\right\}$ a collection of criteria, and $e=\left(e_{1}, e_{2}, \ldots, e_{p}\right)$ the set of DMs. There is a prioritization between the criteria expressed by the linear ordering $C_{1}>C_{2}>\cdots>C_{n}$, indicating that attribute $C_{j}$ has a higher priority than $C_{k}$, if $1 \leq j<k \leq n$ and a prioritization between the experts expressed by the linear ordering $e_{1}>e_{2}>\cdots>e_{p}$, indicating that attribute $e_{j}$ has a higher priority than $e_{k}$, if $1 \leq j<k \leq p$. Suppose that $\widetilde{A}^{k}=\left(\widetilde{a}_{i j}^{k}\right)_{m \times n}$ is the multigranular uncertain linguistic decision matrix provided by $e_{k}$, where $\tilde{a}_{i j}^{k} \in \bar{S}^{\left(g_{k}\right)}$ is the criteria value of $C_{j}$ with respect to alternative $x_{i}$.

In the following, we apply the MULPWA and MULPOWA operators to multiple criteria group decision making based on multigranular uncertain linguistic information. The method involves the following steps.

Step 1. Select the basic linguistic term set $g^{*}$ (in general, the BLT can be chosen by the decision makers directly).

Step 2. Calculate the relative possibility degree of $\tilde{a}_{i j}^{k}$ by utilizing (3) and (5):

$$
P\left(\widetilde{a}_{i j}^{k}\right)=\frac{1}{n(n-1)}\left(\sum_{t=1}^{n} P\left(\widetilde{a}_{i j}^{k} \geq \widetilde{a}_{i t}^{k}\right)+\frac{n}{2}-1\right) .
$$

Step 3. Based on the relative possibility degree, determine the values of $T_{i j}^{k}$ with the following expression:

$$
T_{i j}^{k}= \begin{cases}1, & j=1 \\ \prod_{q=1}^{j-1} P\left(\tilde{a}_{i q}^{k}\right), & j=2, \ldots, n .\end{cases}
$$

Step 4. Utilize the MULPWA operator (12) to aggregate the values $\widetilde{a}_{i j}^{k}$ in the $i$ th row of $\widetilde{A}^{k}$ and get the individual decision results $\vec{a}_{i}^{k}$ corresponding to the alternative $x_{i}$ :

$$
\begin{aligned}
\vec{a}_{i}^{k} & =\operatorname{MUPWA}\left(\tilde{a}_{i 1}^{k}, \tilde{a}_{i 2}^{k}, \ldots, \vec{a}_{i n}^{k}\right) \\
& =\bigoplus_{j=1}^{n}\left(\frac{T_{i j}^{k} \vec{a}_{i j}^{k}}{\sum_{j=1}^{n} T_{i j}^{k}}\right), \quad i=1,2 \ldots, m, k=1,2, \ldots, p .
\end{aligned}
$$

Step 5. Calculate the relative possibility degree of $\tilde{a}_{i}^{k}$, by utilizing (3) and (5) again:

$$
P\left(\vec{a}_{i}^{k}\right)=\frac{1}{p(p-1)}\left(\sum_{t=1}^{p} P\left(\widehat{a}_{i}^{k} \geq \tilde{a}_{i}^{t}\right)+\frac{p}{2}-1\right) .
$$

Step 6. Calculate the values of the values of $T_{i}^{k}$ :

$$
T_{i}^{k}= \begin{cases}1, & k=1 \\ \prod_{q=1}^{k-1} P\left(\widetilde{a}_{i}^{q}\right), & k=2, \ldots, p .\end{cases}
$$

Step 7. Employ the MULPOWA operator (24) to derive the collective decision results $\widetilde{s}_{i}$ of alternative $x_{i}$ :

$$
\begin{aligned}
\tilde{a}_{i} & =\operatorname{MUPOWA}\left(\tilde{a}_{i}^{1}, \tilde{a}_{i}^{1}, \ldots, \tilde{a}_{i}^{p}\right) \\
& =\bigoplus_{k=1}^{p}\left(Q\left(\frac{\sum_{j=1}^{k} T_{i}^{\sigma(k)}}{\sum_{k=1}^{p} T_{i}^{k}}\right)-Q\left(\frac{\sum_{j=1}^{k-1} T_{i}^{\sigma(k)}}{\sum_{k=1}^{p} T_{i}^{k}}\right)\right) \tilde{a}_{i}^{\left(g_{k}\right)} .
\end{aligned}
$$


Step 8. Rank all the alternatives by the possibility degree again.

\section{Numerical Example}

In this section, an example from [40] is used to illustrate the use of the proposed method. To strengthen scientific research and promote the building of teaching body, the school of business in a Chinese university wants to introduce an outstanding professor. The work has been raised great attention from the school, university president $e_{1}$, dean of management school $e_{2}$, and human resource officer $e_{3}$ setting up the panel of decision makers which will take the whole responsibility for this introduction. They made strict evaluation for 5 candidates $x_{i}(i=1,2, \ldots, 5)$ from four aspects, namely, morality $C_{1}$, Tangibles $C_{2}$, Convenience $C_{3}$, and Reliability $C_{4}$. This introduction will be in strict accordance with the principle of combine ability with political integrity. The prioritization relationship for the criteria is as follows, $C_{1}>C_{2}>C_{3}>C_{4}$. Besides, the university president has the absolute priority for decision making; the dean of the management school comes next. Three decision makers evaluate the candidates $x_{i}(i=1,2,3,4,5)$ with respect to the criteria $C_{j}(j=1,2,3,4)$ with linguistic terms sets with different granularities, where $e_{1}$ provides of his judgments uses of linguistic term set with 9 granularities, $S^{(9)}=\left\{s_{0}^{(9)}=\right.$ definitely poor, $s_{1}^{(9)}=$ very poor, $s_{2}^{(9)}=$ poor, $s_{3}^{(9)}=$ slightly poor, $s_{4}^{(9)}=$ medium, $s_{5}^{(9)}=$ slightly poor, $s_{6}^{(9)}=$ good, $s_{7}^{(9)}=$ very good, $s_{8}^{(9)}=$ definitely good $\} ; e_{2}$ provides of his judgments uses of linguistic term set with 7 granularity $S^{(7)}=\left\{s_{0}^{(7)}=\right.$ definitelypoor, $s_{1}^{(7)}=$ verypoor, $s_{2}^{(7)}=$ poor, $s_{3}^{(7)}$ $=$ medium,$s_{4}^{(7)}=$ good, $s_{5}^{(7)}=$ very good, $s_{6}^{(7)}$ $=$ definitely good $\} ; e_{3}$ provides of his judgments uses of linguistic term set with 11 granularities $S^{(11)}=\left\{s_{0}^{(11)}=\right.$ definitely poor, $s_{1}^{(11)}=$ extra poor, $s_{2}^{(11)}=$ very poor, $s_{3}^{(11)}=$ poor, $s_{4}^{(11)}=$ slightly poor, $s_{5}^{(11)}=$ medium, $s_{6}^{(11)}=$ slightly good, $s_{7}^{(11)}=\operatorname{good}, s_{8}^{(11)}=$ very good, $s_{9}^{(11)}=$ extra good, $s_{10}^{(11)}=$ definitely good $\}$. And construct the following multigranular uncertain linguistic decision matrix $\left(\widehat{a}_{i j}^{k}\right)(i=1, \ldots, 5, j=1, \ldots, 4, k=1, \ldots, 3)$, as listed in Table 2.

Step 1. Select the basic linguistic term set. Considering the prioritized levels of decision makers, $S^{(9)}$ used by $e_{1}$ is taken as the BLTS.

Step 2. Calculate the relative possibility degrees of $\tilde{a}_{i j}^{k}(i=$ $1, \ldots, 5, j=1, \ldots, 4, k=1, \ldots, 3)$ by utilizing (31), and the formed possibility degrees matrix is listed in Table 3 .

Step 3. Determine the prioritized levels of $\widehat{a}_{i j}^{k}(i=$ $1, \ldots, 5, j=1, \ldots, 4, k=1, \ldots, 3)$ by utilizing (32), and the prioritized levels of $\vec{a}_{i j}^{k}$ are listed in Table 4 .
TABle 2: Decision matrix.

\begin{tabular}{ccccc}
\hline & $C_{1}$ & $C_{2}$ & $C_{3}$ & $C_{4}$ \\
\hline$x_{1}^{1}$ & {$\left[s_{4}^{(9)}, s_{5}^{(9)}\right]$} & {$\left[s_{5}^{(9)}, s_{6}^{(9)}\right]$} & {$\left[s_{4}^{(9)}, s_{5}^{(9)}\right]$} & {$\left[s_{4}^{(9)}, s_{6}^{(9)}\right]$} \\
$x_{2}^{1}$ & {$\left[s_{7}^{(9)}, s_{8}^{(9)}\right]$} & {$\left[s_{6}^{(9)}, s_{7}^{(9)}\right]$} & {$\left[s_{5}^{(9)}, s_{6}^{(9)}\right]$} & {$\left[s_{5}^{(9)}, s_{6}^{(9)}\right]$} \\
$x_{3}^{1}$ & {$\left[s_{4}^{(9)}, s_{5}^{(9)}\right]$} & {$\left[s_{4}^{(9)}, s_{5}^{(9)}\right]$} & {$\left[s_{6}^{(9)}, s_{7}^{(9)}\right]$} & {$\left[s_{6}^{(9)}, s_{7}^{(9)}\right]$} \\
$x_{4}^{1}$ & {$\left[s_{5}^{(9)}, s_{6}^{(9)}\right]$} & {$\left[s_{3}^{(9)}, s_{5}^{(9)}\right]$} & {$\left[s_{4}^{(9)}, s_{6}^{(9)}\right]$} & {$\left[s_{6}^{(9)}, s_{7}^{(9)}\right]$} \\
$x_{5}^{1}$ & {$\left[s_{4}^{(9)}, s_{6}^{(9)}\right]$} & {$\left[s_{6}^{(9)}, s_{8}^{(9)}\right]$} & {$\left[s_{5}^{(9)}, s_{7}^{(9)}\right]$} & {$\left[s_{5}^{(9)}, s_{6}^{(9)}\right]$} \\
\hline$x_{1}^{2}$ & {$\left[s_{3}^{(7)}, s_{4}^{(7)}\right]$} & {$\left[s_{3}^{(7)}, s_{5}^{(7)}\right]$} & {$\left[s_{4}^{(7)}, s_{5}^{(7)}\right]$} & {$\left[s_{3}^{(7)}, s_{5}^{(7)}\right]$} \\
$x_{2}^{2}$ & {$\left[s_{4}^{(7)}, s_{5}^{(7)}\right]$} & {$\left[s_{3}^{(7)}, s_{4}^{(7)}\right]$} & {$\left[s_{3}^{(7)}, s_{5}^{(7)}\right]$} & {$\left[s_{3}^{(7)}, s_{5}^{(7)}\right]$} \\
$x_{3}^{2}$ & {$\left[s_{2}^{(7)}, s_{3}^{(7)}\right]$} & {$\left[s_{2}^{(7)}, s_{4}^{(7)}\right]$} & {$\left[s_{3}^{(7)}, s_{5}^{(7)}\right]$} & {$\left[s_{4}^{(7)}, s_{5}^{(7)}\right]$} \\
$x_{4}^{2}$ & {$\left[s_{4}^{(7)}, s_{5}^{(7)}\right]$} & {$\left[s_{3}^{(7)}, s_{4}^{(7)}\right]$} & {$\left[s_{2}^{(7)}, s_{3}^{(7)}\right]$} & {$\left[s_{5}^{(7)}, s_{6}^{(7)}\right]$} \\
$x_{5}^{2}$ & {$\left[s_{3}^{(7)}, s_{4}^{(7)}\right]$} & {$\left[s_{5}^{(7)}, s_{6}^{(7)}\right]$} & {$\left[s_{4}^{(7)}, s_{6}^{(7)}\right]$} & {$\left[s_{3}^{(7)}, s_{5}^{(7)}\right]$} \\
\hline$x_{1}^{3}$ & {$\left[s_{4}^{(11)}, s_{5}^{(11)}\right]$} & {$\left[s_{6}^{(11)}, s_{7}^{(11)}\right]$} & {$\left[s_{5}^{(11)}, s_{6}^{(11)}\right]$} & {$\left[s_{6}^{(11)}, s_{8}^{(11)}\right]$} \\
$x_{2}^{3}$ & {$\left[s_{8}^{(11)}, s_{10}^{(11)}\right]$} & {$\left[s_{7}^{(11)}, s_{9}^{(11)}\right]$} & {$\left[s_{6}^{(11)}, s_{7}^{(11)}\right]$} & {$\left[s_{6}^{(11)}, s_{9}^{(11)}\right]$} \\
$x_{3}^{3}$ & {$\left[s_{6}^{(11)}, s_{7}^{(11)}\right]$} & {$\left[s_{5}^{(11)}, s_{6}^{(11)}\right]$} & {$\left[s_{8}^{(11)}, s_{10}^{(11)}\right]$} & {$\left[s_{9}^{(11)}, s_{10}^{(11)}\right]$} \\
$x_{4}^{3}$ & {$\left[s_{9}^{(11)}, s_{10}^{(11)}\right]$} & {$\left[s_{6}^{(11)}, s_{9}^{(11)}\right]$} & {$\left[s_{6}^{(11)}, s_{7}^{(11)}\right]$} & {$\left[s_{5}^{(11)}, s_{8}^{(11)}\right]$} \\
$x_{5}^{3}$ & {$\left[s_{6}^{(11)}, s_{7}^{(11)}\right]$} & {$\left[s_{7}^{(11)}, s_{9}^{(11)}\right]$} & {$\left[s_{9}^{(11)}, s_{10}^{(11)}\right]$} & {$\left[s_{7}^{(11)}, s_{9}^{(11)}\right]$} \\
\hline
\end{tabular}

TABLE 3: Possibility degree of $\tilde{a}_{i j}^{k}$.

\begin{tabular}{ccccc}
\hline & $C_{1}$ & $C_{2}$ & $C_{3}$ & $C_{4}$ \\
\hline$x_{1}^{1}$ & 0.1944 & 0.3473 & 0.1944 & 0.2639 \\
$x_{2}^{1}$ & 0.375 & 0.2916 & 0.1667 & 0.1667 \\
$x_{3}^{1}$ & 0.1667 & 0.1667 & 0.3333 & 0.3333 \\
$x_{4}^{1}$ & 0.2639 & 0.1458 & 0.2153 & 0.375 \\
$x_{5}^{1}$ & 0.1736 & 0.3542 & 0.2639 & 0.2083 \\
\hline$x_{1}^{2}$ & 0.1805 & 0.236 & 0.3195 & 0.264 \\
$x_{2}^{2}$ & 0.3195 & 0.1805 & 0.25 & 0.25 \\
$x_{3}^{2}$ & 0.1528 & 0.2014 & 0.2986 & 0.3472 \\
$x_{4}^{2}$ & 0.2917 & 0.2083 & 0.125 & 0.375 \\
$x_{5}^{2}$ & 0.1528 & 0.2986 & 0.3264 & 0.2222 \\
\hline$x_{1}^{3}$ & 0.125 & 0.3194 & 0.2083 & 0.3473 \\
$x_{2}^{3}$ & 0.3375 & 0.2792 & 0.1458 & 0.2375 \\
$x_{3}^{3}$ & 0.2083 & 0.125 & 0.3194 & 0.3473 \\
$x_{4}^{3}$ & 0.375 & 0.2431 & 0.1875 & 0.1944 \\
$x_{5}^{3}$ & 0.125 & 0.25 & 0.3542 & 0.2708 \\
\hline
\end{tabular}

Step 4. Derive the individual decision results $\widetilde{a}_{i}^{k}$ corresponding to the alternative $x_{i}$ by utilizing (33):

$$
\begin{aligned}
\tilde{a}_{1}^{1} & =\operatorname{MULPWA}\left(\tilde{a}_{11}^{1}, \tilde{a}_{12}^{1}, \tilde{a}_{13}^{1}, \tilde{a}_{14}^{1}\right) \\
& =\frac{1}{1.275}\left[s_{4}^{(9)}, s_{5}^{(9)}\right] \oplus \frac{0.1944}{1.275}\left[s_{5}^{(9)}, s_{6}^{(9)}\right]
\end{aligned}
$$


TABle 4: Prioritized level of $\widetilde{a}_{i j}^{k}$.

\begin{tabular}{cccccc}
\hline & $T_{1}$ & $T_{2}$ & $T_{3}$ & $T_{4}$ & $\sum_{j=1}^{4} T_{j}$ \\
\hline$x_{1}^{1}$ & 1 & 0.1944 & 0.0675 & 0.0131 & 1.275 \\
$x_{2}^{1}$ & 1 & 0.375 & 0.1094 & 0.0182 & 1.5026 \\
$x_{3}^{1}$ & 1 & 0.1667 & 0.0278 & 0.0093 & 1.2038 \\
$x_{4}^{1}$ & 1 & 0.2639 & 0.0385 & 0.0083 & 1.3107 \\
$x_{5}^{1}$ & 1 & 0.1736 & 0.0615 & 0.0162 & 1.2513 \\
\hline$x_{1}^{2}$ & 1 & 0.1805 & 0.0426 & 0.0136 & 1.2367 \\
$x_{2}^{2}$ & 1 & 0.3195 & 0.0577 & 0.0144 & 1.3916 \\
$x_{3}^{2}$ & 1 & 0.1528 & 0.0308 & 0.0092 & 1.1928 \\
$x_{4}^{2}$ & 1 & 0.2917 & 0.0608 & 0.0076 & 1.3601 \\
$x_{5}^{2}$ & 1 & 0.1528 & 0.0456 & 0.0149 & 1.2133 \\
\hline$x_{1}^{3}$ & 1 & 0.125 & 0.0399 & 0.0083 & 1.1732 \\
$x_{2}^{3}$ & 1 & 0.3375 & 0.0942 & 0.0137 & 1.4454 \\
$x_{3}^{3}$ & 1 & 0.2083 & 0.026 & 0.0083 & 1.2426 \\
$x_{4}^{3}$ & 1 & 0.375 & 0.0912 & 0.0171 & 1.4833 \\
$x_{5}^{3}$ & 1 & 0.125 & 0.0313 & 0.0111 & 1.1674 \\
\hline
\end{tabular}

TABLE 5: Individual decision results.

\begin{tabular}{llll}
\hline & $e_{1}$ & $e_{2}$ & \multicolumn{1}{c}{$e_{3}$} \\
\hline$x_{1}$ & {$\left[s_{4.1525}^{(9)}, s_{5.1628}^{(9)}\right]$} & {$\left[s_{3.0344}^{(7)}, s_{4.1914}^{(7)}\right]$} & {$\left[s_{4.2612}^{(11)}, s_{5.2683}^{(11)}\right]$} \\
$x_{2}$ & {$\left[s_{6.5006}^{(9)}, s_{7.5806}^{(9)}\right]$} & {$\left[s_{3.7186}^{(7)}, s_{4.7704}^{(7)}\right]$} & {$\left[s_{7.6171}^{(11)}, s_{9.5614}^{(11)}\right]$} \\
$x_{3}$ & {$\left[s_{4.0616}^{(9)}, s_{5.0616}^{(9)}\right]$} & {$\left[s_{2.0412}^{(7)}, s_{3.1951}^{(7)}\right]$} & {$\left[s_{5.8943}^{(11)}, s_{6.9152}^{(11)}\right]$} \\
$x_{4}$ & {$\left[s_{4.5743}^{(9)}, s_{5.805}^{(9)}\right]$} & {$\left[s_{3.7107}^{(7)}, s_{4.7017}^{(7)}\right]$} & {$\left[s_{8.3321}^{(11)}, s_{9.5397}^{(11)}\right]$} \\
$x_{5}$ & {$\left[s_{4.3395}^{(9)}, s_{6.3266}^{(9)}\right]$} & {$\left[s_{3.2894}^{(7)}, s_{4.3393}^{(7)}\right]$} & {$\left[s_{6.1963}^{(11)}, s_{7.3136}^{(11)}\right]$} \\
\hline
\end{tabular}

$$
\begin{aligned}
& \oplus \frac{0.0675}{1.275}\left[s_{4}^{(9)}, s_{5}^{(9)}\right] \oplus \frac{0.0131}{1.275}\left[s_{4}^{(9)}, s_{6}^{(9)}\right] \\
= & \frac{1}{1.275}\left[s_{4}^{(9)}, s_{5}^{(9)}\right] \oplus \frac{0.1944}{1.275}\left[s_{5}^{(9)}, s_{6}^{(9)}\right] \oplus \frac{0.0675}{1.275}\left[s_{4}^{(9)}, s_{5}^{(9)}\right] \\
& \oplus \frac{0.0131}{1.275}\left[s_{4}^{(9)}, s_{6}^{(9)}\right]=\left[s_{4.1525}^{(9)}, s_{5.1628}^{(9)}\right] .
\end{aligned}
$$

Note that since the aggregation is from the same linguistic term set here, thence the MULPWA operator is essentially ULPWA operator here. Similarly, we can get all individual decision results listed in Table 5.

Step 5. Determine the relative possibility degree of $\tilde{a}_{i}^{k}(i=$ $1, \ldots, 5, k=1, \ldots, 3)$ by utilizing (34), and the possibility degree of $\tilde{a}_{i}^{k}$ is listed in Table 6 .

Step 6. Derive the prioritized levels of $\tilde{a}_{i}^{k}(i=1, \ldots, 5, k=$ $1, \ldots, 3)$ by utilizing (35), the prioritized levels of $\tilde{a}_{i}^{k}$ are listed in Table 7.
TABLe 6: Possibility degrees of individual decision results.

\begin{tabular}{lccc}
\hline & $e_{1}$ & $e_{2}$ & $e_{3}$ \\
\hline$x_{1}$ & 0.4006 & 0.4151 & 0.1843 \\
$x_{2}$ & 0.4303 & 0.1817 & 0.388 \\
$x_{3}$ & 0.3521 & 0.1797 & 0.4682 \\
$x_{4}$ & 0.2227 & 0.2773 & 0.5 \\
$x_{5}$ & 0.3414 & 0.2981 & 0.3605 \\
\hline
\end{tabular}

TABLe 7: Prioritized levels of individual decision results.

\begin{tabular}{ccccc}
\hline & $T_{1}$ & $T_{2}$ & $T_{3}$ & $\sum_{j=1}^{3} T_{j}$ \\
\hline$x_{1}$ & 1 & 0.4006 & 0.1663 & 1.5669 \\
$x_{2}$ & 1 & 0.4303 & 0.0782 & 1.5085 \\
$x_{3}$ & 1 & 0.3521 & 0.0633 & 1.4154 \\
$x_{4}$ & 1 & 0.2227 & 0.0618 & 1.2845 \\
$x_{5}$ & 1 & 0.3414 & 0.1018 & 1.4432 \\
\hline
\end{tabular}

Step 7. Utilize the MULPOWA operator (36) to derive the collective decision results of alternatives $x_{i}(i=1,2, \ldots, 5)$ :

$$
\begin{aligned}
\tilde{a}_{1}= & \operatorname{MULPOWA}\left(\tilde{a}_{1}^{1}, \tilde{a}_{1}^{2}, \tilde{a}_{1}^{3}\right) \\
= & \left(Q\left(\frac{0.4006}{1.5669}\right)-Q\left(\frac{0}{1.5669}\right)\right)\left[s_{3.0344}^{(7)}, s_{4.1914}^{(7)}\right] \\
& \oplus\left(Q\left(\frac{0.4006+1}{1.5669}\right)-Q\left(\frac{0.4006}{1.5669}\right)\right)\left[s_{4.1525}^{(9)}, s_{5.1628}^{(9)}\right] \\
& \oplus\left(Q\left(\frac{1.5669}{1.5669}\right)-Q\left(\frac{0.4006+1}{1.5669}\right)\right)\left[s_{4.2612}^{(11)}, s_{5.2683}^{(11)}\right] \\
= & 0.065\left[s_{4.046}^{(9)}, s_{5.589}^{(9)}\right] \oplus 0.734\left[s_{4.1525}^{(9)}, s_{5.1628}^{(9)}\right] \\
& \oplus 0.201\left[s_{3.41}^{(11)}, s_{4.21}^{(11)}\right]=\left[s_{4}^{(9)}, s_{5}^{(9)}\right] .
\end{aligned}
$$

Similarly, we can get the rest collective decision results.

$$
\begin{aligned}
& \tilde{a}_{2}=\operatorname{MULPOWA}\left(\tilde{x}_{2}^{1}, \tilde{x}_{2}^{2}, \tilde{x}_{2}^{3}\right)=\left[s_{5.75}^{(9)}, s_{6.99}^{(9)}\right], \\
& \tilde{a}_{3}=\operatorname{MULPOWA}\left(\tilde{x}_{3}^{1}, \tilde{x}_{3}^{2}, \tilde{x}_{3}^{3}\right)=\left[s_{3.48}^{(9)}, s_{4.71}^{(9)}\right], \\
& \tilde{a}_{4}=\operatorname{MULPOWA}\left(\tilde{x}_{4}^{1}, \tilde{x}_{4}^{2}, \tilde{x}_{4}^{3}\right)=\left[s_{4.59}^{(9)}, s_{5.84}^{(9)}\right], \\
& \tilde{a}_{5}=\operatorname{MULPOWA}\left(\tilde{x}_{5}^{1}, \tilde{x}_{5}^{2}, \tilde{x}_{5}^{3}\right)=\left[s_{4.36}^{(9)}, s_{6.10}^{(9)}\right] .
\end{aligned}
$$


Step 8. Rank all the alternatives by using the possibility degrees

$$
\begin{aligned}
x_{2} & =\left[s_{5.75}^{(9)}, s_{6.99}^{(9)}\right]>x_{4}=\left[s_{4.59}^{(9)}, s_{5.84}^{(9)}\right] \succ x_{5} \\
& =\left[s_{4.36}^{(9)}, s_{6.10}^{(9)}\right]>x_{1}=\left[s_{4}^{(9)}, s_{5}^{(9)}\right] \succ x_{3}=\left[s_{3.48}^{(9)}, s_{4.71}^{(9)}\right] .
\end{aligned}
$$

Thus the best alternative is $x_{2}$.

If the criteria or the decision makers are at the same priority level and the values of uncertain linguistic variables derived from the same linguistic terms set, then the above operators are reduced to the traditional uncertain linguistic aggregation operators proposed by $\mathrm{Xu}[13]$. However, there are different priority levels among these four criteria and three decision makers. For example, the candidate is very hard to be selected when he received a poor evaluation from the manager. From another point of view, if a candidate has a bad work attitude, then he is impossible to select no matter how good of performance evaluation he has received on capacity for work, leadership, and learning skill. Therefore, we must consider the prioritization among the criteria or the decision makers. What is more, if we use the traditional uncertain linguistic aggregation operators to aggregate the uncertain linguistic information derived from different linguistic terms sets, the transform processes with tedious computation are inevitable. To deal with such situations, the proposed operator is an effective tool. From the above analysis, the main advantages of the proposed operators are not only due to the fact that our operators can aggregate directly the multigranularity linguistic information without tedious calculation to unify the multigranular uncertain linguistic information, but also due to the consideration of the prioritized relationships between the decision elements, which makes it more feasible and practical.

\section{Conclusion}

In this paper, some operational laws of the multigranular uncertain linguistic variables are first defined. Based on prioritized aggregation operator, we developed some multigranular uncertain linguistic prioritized aggregation operators, such as multigranular uncertain linguistic prioritized weighted averaging operator multigranular uncertain linguistic prioritized ordered weighted average operator. The distinct characteristics of these proposed operators are that they can aggregate directly the multigranular uncertain linguistic information and take into account prioritization among the arguments. Then, we have utilized these operators to develop an approach to solve the multigranular uncertain linguistic multiple criteria group decision-making problems in which the criteria are in different priority levels. Finally, a practical example about talent introduction is given to verify the developed approach and to demonstrate its practicality and effectiveness. It is noted that the proposed multigranular uncertain linguistic prioritized aggregation operators are based on the uniform and symmetrical linguistic terms sets. However, in some real-life situations, decision makers may provide their judgments with unbalanced linguistic term sets $[28,33-35]$, which are not uniformly and symmetrically distributed, to emphasize the different discrimination levels on both sides of mid linguistic term. In the future, we will investigate the multigranular uncertain linguistic prioritized operators to the unbalanced linguistic contexts.

\section{Acknowledgments}

The authors are very grateful to the editor, Professor Frank Werner, and the anonymous referees for their insightful and constructive comments and suggestions which have helped to improve the paper. This work was supported in part by the National Natural Science Funds of China (Projects nos. 71272191 and 71072085) and the Graduate Innovation Funds of Heilongjiang Province of China (Project no.YJSCX2011003HLJ).

\section{References}

[1] R. Degani and G. Bortolan, "The problem of linguistic approximation in clinical decision making," International Journal of Approximate Reasoning, vol. 2, no. 2, pp. 143-162, 1988.

[2] M. Delgado, J. L. Verdegay, and M. A. Vila, "On aggregation operations of linguistic labels," International Journal of Intelligent Systems, vol. 8, no. 3, pp. 351-370, 1993.

[3] G. Bordogna, M. Fedrizzi, and G. Pasi, "A linguistic modeling of consensus in group decision making based on OWA operators," IEEE Transactions on Systems, Man, and Cybernetics Part A, vol. 27, no. 1, pp. 126-132, 1997.

[4] F. Herrera and E. Herrera-Viedma, "Linguistic decision analysis: steps for solving decision problems under linguistic information," Fuzzy Sets and Systems, vol. 115, no. 1, pp. 67-82, 2000.

[5] F. Herrera, S. Alonso, F. Chiclana, and E. Herrera-Viedma, "Computing with words in decision making: foundations, trends and prospects," Fuzzy Optimization and Decision Making, vol. 8, no. 4, pp. 337-364, 2009.

[6] J. F. Pang and J. Y. Liang, "Evaluation of the results of multiattribute group decision-making with linguistic information," Omega, vol. 40, no. 3, pp. 294-301, 2012.

[7] L. Martínez and F. Herrera, "An overview on the 2-tuple linguistic model for computing with words in decision making: extensions, applications and challenges," Information Sciences, vol. 207, pp. 1-18, 2012.

[8] F. Herrera and L. Martinez, "A 2-tuple fuzzy linguistic representation model for computing with words," IEEE Transactions on Fuzzy Systems, vol. 8, no. 6, pp. 746-752, 2000.

[9] Z.S. Xu, "EOWA and EOWG operators for aggregating linguistic labels based on linguistic preference relations," International Journal of Uncertainty, Fuzziness and Knowlege-Based Systems, vol. 12, no. 6, pp. 791-810, 2004.

[10] Z. S. Xu, "A method based on linguistic aggregation operators for group decision making with linguistic preference relations," Information Sciences, vol. 166, no. 1-4, pp. 19-30, 2004.

[11] Y. C. Dong, Y. F. Xu, and S. Yu, "Linguistic multiperson decision making based on the use of multiple preference relations," Fuzzy Sets and Systems, vol. 160, no. 5, pp. 603-623, 2009. 
[12] Y. C. Dong, Y. F. Xu, H. Y. Li, and B. Feng, “The OWA-based consensus operator under linguistic representation models using position indexes," European Journal of Operational Research, vol. 203, no. 2, pp. 455-463, 2010.

[13] Z. S. Xu, "Uncertain linguistic aggregation operators based approach to multiple attribute group decision making under uncertain linguistic environment," Information Sciences, vol. 168, no. 1-4, pp. 171-184, 2004.

[14] Z. S. Xu, "Induced uncertain linguistic OWA operators applied to group decision making," Information Fusion, vol. 7, no. 2, pp. 231-238, 2006.

[15] Z. S. Xu, "An approach based on the uncertain LOWG and induced uncertain LOWG operators to group decision making with uncertain multiplicative linguistic preference relations," Decision Support Systems, vol. 41, no. 2, pp. 488-499, 2006.

[16] Z. S. Xu, "Correlated linguistic information aggregation," International Journal of Uncertainty, Fuzziness and Knowlege-Based Systems, vol. 17, no. 5, pp. 633-647, 2009.

[17] G. W. Wei, "Uncertain linguistic hybrid geometric mean operator and its application to group decision making under uncertain linguistic environment," International Journal of Uncertainty, Fuzziness and Knowlege-Based Systems, vol. 17, no. 2, pp. 251-267, 2009.

[18] J. H. Park, M. G. Gwak, and Y. C. Kwun, "Uncertain linguistic harmonic mean operators and their applications to multiple attribute group decision making," Computing, vol. 93, no. 1, pp. 47-64, 2011.

[19] H. M. Zhang, "The multiattribute group decision making method based on aggregation operators with interval-valued 2-tuple linguistic information," Mathematical and Computer Modelling, vol. 56, no. 1-2, pp. 27-35, 2012.

[20] S.-M. Chen and L.-W. Lee, "Autocratic decision making using group recommendations based on the ILLOWA operator and likelihood-based comparison relations," IEEE Transactions on Systems, Man, and Cybernetics Part A, vol. 42, no. 1, pp. 115-129, 2012.

[21] S.-M. Chen, L.-W. Lee, S.-W. Yang, and T.-W. Sheu, "Adaptive consensus support model for group decision making systems," Expert Systems with Applications, vol. 39, no. 16, pp. 1258012588, 2012.

[22] F. Herrera, E. Herrera-Viedma, and L. Martínez, "A fusion approach for managing multi-granularity linguistic term sets in decision making," Fuzzy Sets and Systems, vol. 114, no. 1, pp. 43$58,2000$.

[23] F. Herrera and L. Martínez, "A model based on linguistic 2tuples for dealing with multigranular hierarchical linguistic contexts in multi-expert decision-making," IEEE Transactions on Systems, Man, and Cybernetics Part B, vol. 31, no. 2, pp. 227234, 2001.

[24] E. Herrera-Viedma, L. Martínez, F. Mata, and F. Chiclana, "A consensus support system model for group decision-making problems with multigranular linguistic preference relations," IEEE Transactions on Fuzzy Systems, vol. 13, no. 5, pp. 644-658, 2005.

[25] Z. F. Chen and D. Ben-Arieh, "On the fusion of multigranularity linguistic label sets in group decision making," Computers and Industrial Engineering, vol. 51, no. 3, pp. 526541, 2006.

[26] Y. P. Jiang, Z. P. Fan, and J. Ma, "A method for group decision making with multi-granularity linguistic assessment information," Information Sciences, vol. 178, no. 4, pp. 1098-1109, 2008.
[27] F. Mata, L. Martínez, and E. Herrera-Viedma, "An adaptive consensus support model for group decision-making problems in a multigranular fuzzy linguistic context," IEEE Transactions on Fuzzy Systems, vol. 17, no. 2, pp. 279-290, 2009.

[28] Z. S. Xu, "An interactive approach to multiple attribute group decision making with multigranular uncertain linguistic information," Group Decision and Negotiation, vol. 18, no. 2, pp. 119145, 2009.

[29] X. H. Yu, Z. S. Xu, and X. M. Zhang, "Uniformization of multigranular linguistic labels and their application to group decision making," Journal of Systems Science and Systems Engineering, vol. 19, no. 3, pp. 257-276, 2010.

[30] Z. P. Fan and Y. Liu, "A method for group decision-making based on multi-granularity uncertain linguistic information," Expert Systems with Applications, vol. 37, no. 5, pp. 4000-4008, 2010.

[31] C. Y. Gao and D. H. Peng, "Consolidating SWOT analysis with nonhomogeneous uncertain preference information," Knowledge-Based Systems, vol. 24, no. 6, pp. 796-808, 2011.

[32] Z. Zhang and C. H. Guo, "A method for multi-granularity uncertain linguistic group decision making with incomplete weight information," Knowledge-Based Systems, vol. 26, pp. 111119, 2012.

[33] E. Herrera-Viedma and A. G. López-Herrera, "A model of an information retrieval system with unbalanced fuzzy linguistic information," International Journal of Intelligent Systems, vol. 22, no. 11, pp. 1197-1214, 2007.

[34] F. Herrera, E. Herrera-Viedma, and L. Martínez, "A fuzzy linguistic methodology to deal with unbalanced linguistic term sets," IEEE Transactions on Fuzzy Systems, vol. 16, no. 2, pp. 354370, 2008.

[35] F. J. Cabrerizo, S. Alonso, and E. Herrera-Viedma, "A consensus model for group decision making problems with unbalanced fuzzy linguistic information," International Journal of Information Technology and Decision Making, vol. 8, no. 1, pp. 109-131, 2009.

[36] R. R. Yager, "Modeling prioritized multicriteria decision making," IEEE Transactions on Systems, Man, and Cybernetics Part B, vol. 34, no. 6, pp. 2396-2404, 2004.

[37] R. R. Yager, “Prioritized aggregation operators," International Journal of Approximate Reasoning, vol. 48, no. 1, pp. 263-274, 2008.

[38] R. R. Yager, "Prioritized OWA aggregation," Fuzzy Optimization and Decision Making, vol. 8, no. 3, pp. 245-262, 2009.

[39] R. R. Yager, G. Gumrah, and M. Z. Reformat, "Using a web personal evaluation tool-PET for lexicographic multi-criteria service selection," Knowledge-Based Systems, vol. 24, no. 7, pp. 929-942, 2011.

[40] D. J. Yu, Y. Y. Wu, and T. Lu, "Interval-valued intuitionistic fuzzy prioritized operators and their application in group decision making," Knowledge-Based Systems, vol. 30, pp. 57-66, 2012.

[41] R. R. Yager, “On prioritized multiple-criteria aggregation," IEEE Transactions on Systems, Man, and Cybernetics Part B, vol. 42, no. 5, pp. 1297-1305, 2012.

[42] Z. S. Xu, "Algorithm for priority of fuzzy complementary judgment matrix," Journal of System Engineering, vol. 16, no. 4, pp. 311-314, 2001.

[43] R. R. Yager, "On ordered weighted averaging aggregation operators in multicriteria decisionmaking," IEEE Transactions on Systems, Man, and Cybernetics, vol. 18, no. 1, pp. 183-190, 1988. 
[44] R. R. Yager, "Quantifier guided aggregation using OWA operators," International Journal of Intelligent Systems, vol. 11, no. 1, pp. 49-73, 1996. 


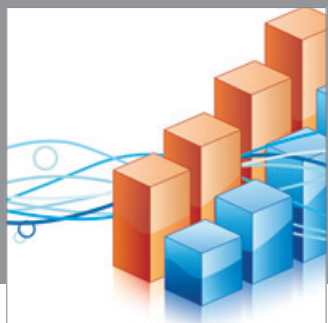

Advances in

Operations Research

mansans

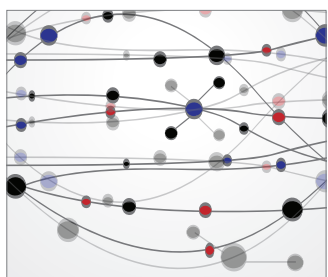

The Scientific World Journal
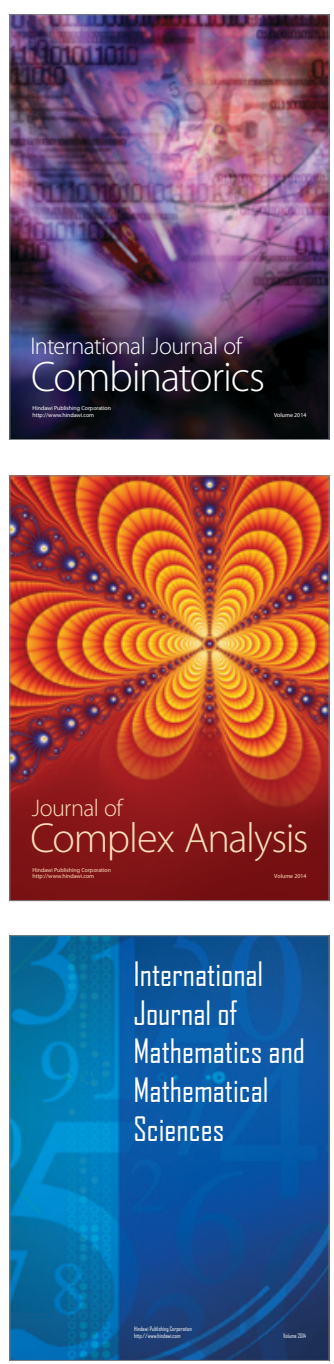
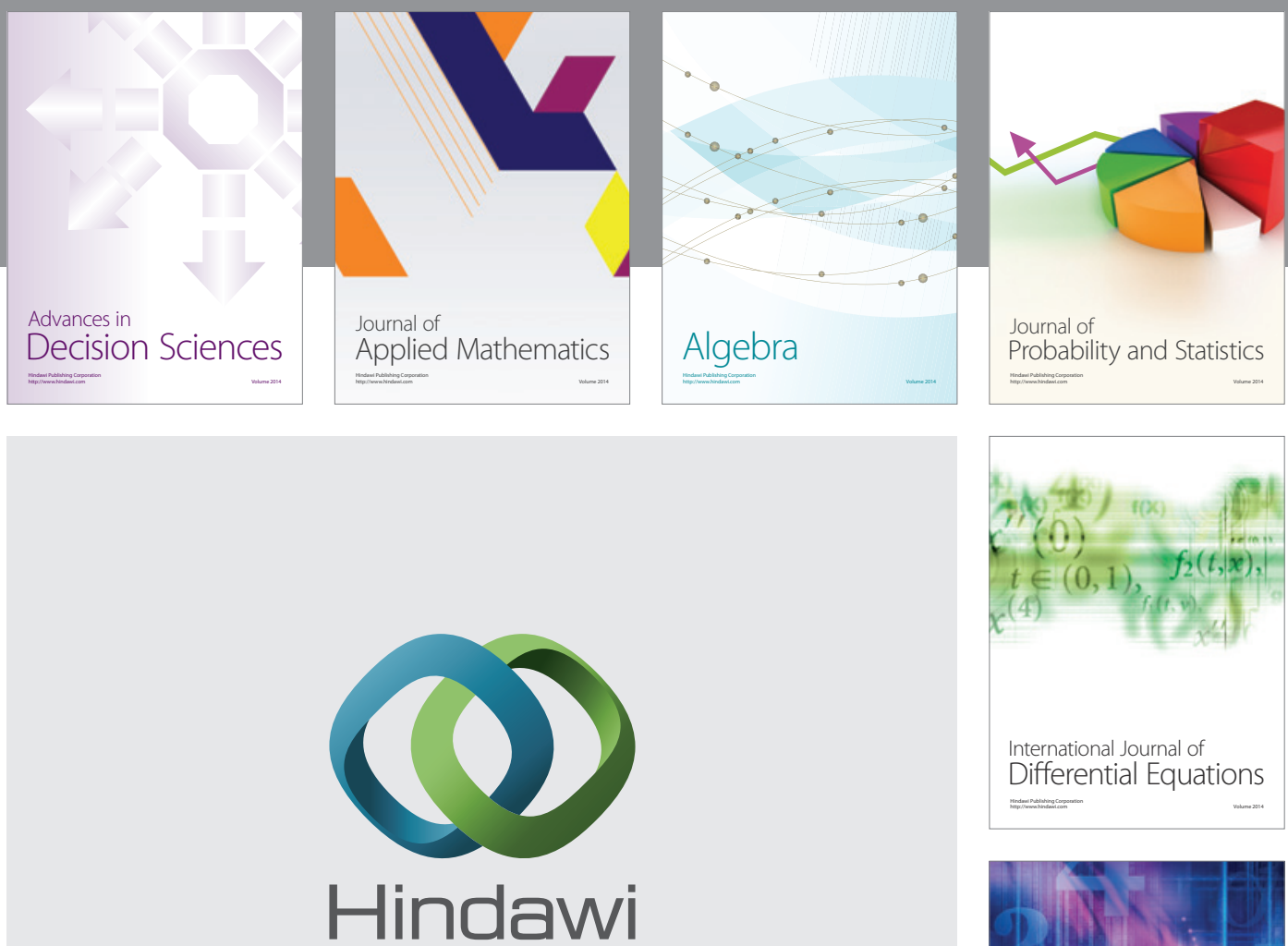

Submit your manuscripts at http://www.hindawi.com
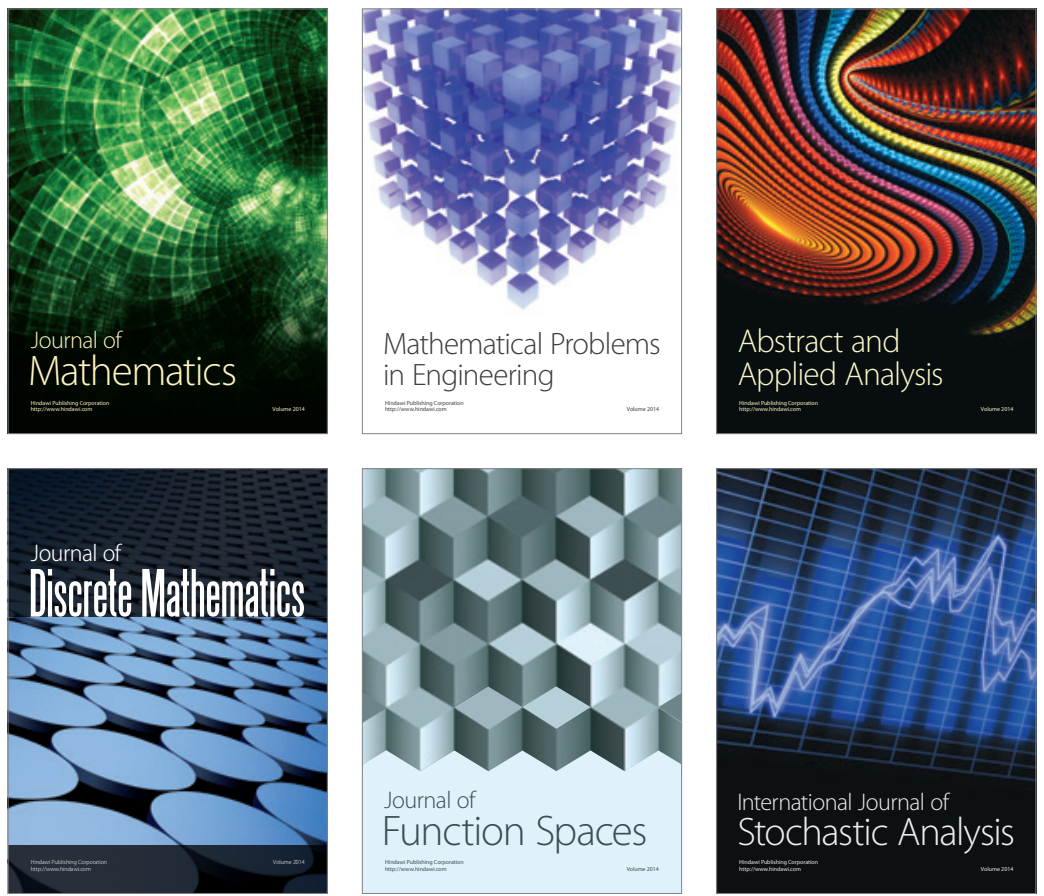

Journal of

Function Spaces

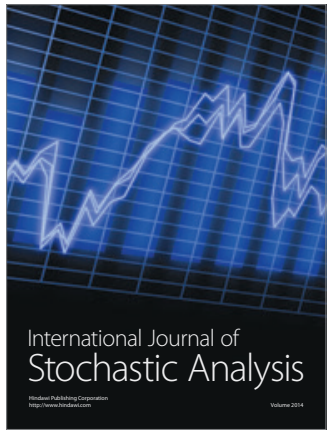

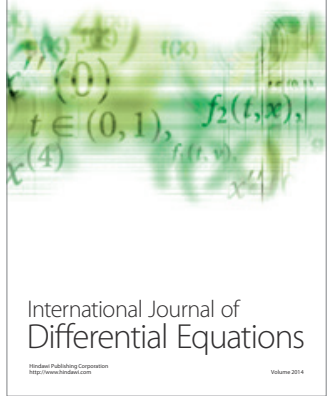
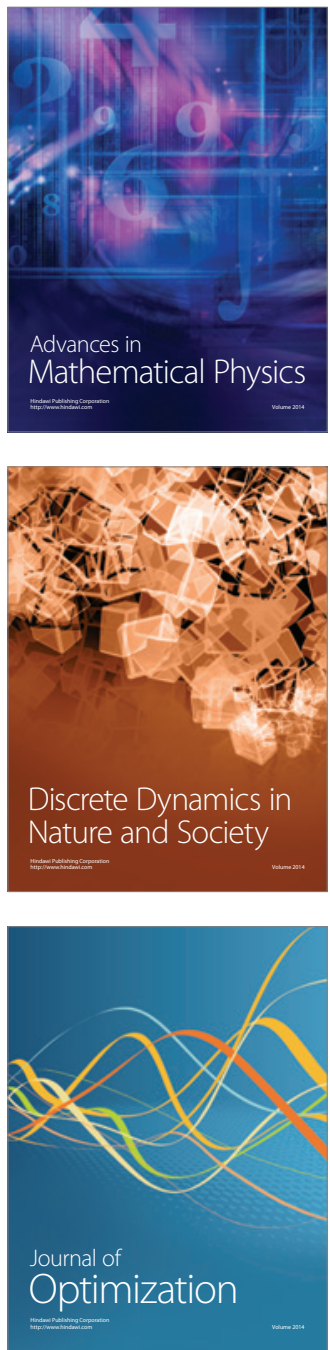